\title{
Hybrid Nanomaterials Based on Iron Oxide Nanoparticles and Mesoporous Silica Nanoparticles: Overcoming Challenges in Current Cancer Treatments
}

\author{
Merlis P. Alvarez-Berríos, ${ }^{1}$ Naisha Sosa-Cintron, ${ }^{1}$ Mariel Rodriguez-Lugo, ${ }^{1}$ \\ Ridhima Juneja, ${ }^{2}$ and Juan L. Vivero-Escoto ${ }^{2,3}$ \\ ${ }^{1}$ Department of Science and Technology, Inter American University of Puerto Rico, Ponce Campus, Ponce, PR 00716, USA \\ ${ }^{2}$ Department of Chemistry, The University of North Carolina at Charlotte, Charlotte, NC 28223, USA \\ ${ }^{3}$ The Center for Biomedical Engineering and Science, The University of North Carolina at Charlotte, Charlotte, NC 28223, USA
}

Correspondence should be addressed to Merlis P. Alvarez-Berríos; mpalvare@ponce.inter.edu

Received 26 February 2016; Accepted 11 April 2016

Academic Editor: Dennis Douroumis

Copyright (C) 2016 Merlis P. Alvarez-Berríos et al. This is an open access article distributed under the Creative Commons Attribution License, which permits unrestricted use, distribution, and reproduction in any medium, provided the original work is properly cited.

\begin{abstract}
The current approaches used for the treatment of cancer face some clinical limitations such as induction of severe side effects, multidrug resistance (MDR), and low specificity toward metastatic cancer cells. Hybrid nanomaterials hold a great potential to overcome all these challenges. Among hybrid nanoparticles, those based on mesoporous silica and iron oxide nanoparticles (MSNs and IONPs) have gained a privileged place in the biomedical field because of their outstanding properties. There are many studies demonstrating their effectiveness as drug delivery systems, nanoheaters, and imaging contrast agents. This review summarizes the advances related to the utilization of IONPs and MSNs for reducing side effects, overcoming MDR, and inhibiting metastasis. Furthermore, we give a future perspective of the clinical application of these technologies.
\end{abstract}

\section{Introduction}

Cancer is the second cause of death in the United States and will be responsible for 595,690 deaths in 2016 [1]. The diagnosis and treatment of this deadly disease is challenging due to clinical limitations of traditional approaches such as surgery, radiotherapy, and chemotherapy [2-4]. For decades, chemotherapy has been regarded as the standard form of clinical therapy for most types of cancer. However, the induction of severe side effects and multidrug resistance (MDR) limit the use and efficacy of this antineoplastic therapy $[2,5]$. Moreover, due to the poor therapeutic outcome reached in many solid tumors, cancer cells have the ability to travel from their primary tumor and to reestablish in other organs (metastasis). The formation of secondary tumors in distant organs impacts negatively the survival of cancer patients [6]. For these reasons, detrimental side effects, MDR, and metastasis are the greatest challenges in cancer treatment today.
The utilization of hybrid nanomaterials, which are composed of organic and inorganic materials, has great potential to overcome some of the major issues related to the treatment of cancer. Several publications have shown the application of hybrid nanoparticles for cancer treatment $[7,8]$. They have been used as drug delivery systems, nanoheaters, and MRI contrast agents without inducing significant side effects [710]. In addition, nanoparticles can be targeted to tumors by a passive mechanism known as enhanced permeability and retention (EPR) effect and an active approach using targeting agents that allow the specific interaction of the target-modified nanomaterial with cancer cells [10]. While the EPR effect is the result of the leaky vasculature and impaired lymphatic function of tumor tissue, active targeting can be achieved by the conjugation of specific molecules or antibodies onto the surface of nanoparticles to render the ability of recognizing specific protein receptors overexpressed in cancer cells $[10,11]$. This could result in the accumulation 
of nanoparticles in the tumor tissue and/or in cellular uptake, allowing the intracellular delivery of therapeutic agents.

The use of different types of hybrid nanomaterials based on iron oxide and mesoporous silica nanoparticles (IONPs and MSNs) have been widely explored in biomedical research with promising results for cancer therapy [10, 12-15]. This review summarizes the advances related to the use of IONPs and MSNs for reducing side effects, overcoming MDR and inhibiting metastasis. Furthermore, we give a future perspective about the translation of these technologies to a clinically available treatment.

\section{Iron Oxide Nanoparticles}

For more than a decade, IONPs have gained significant interest, in particular for their application in the biomedical field. This growing interest is due to their exclusive properties such as their ability to respond to magnetic fields (in magnetic field gradients), particle rotation (in alternating magnetic fields), or internal dipole rotation (in alternating magnetic field) [10]. As a result, IONPs can be directed to specific sites using a magnetic field or dissipate magnetic energy in the form of heat when an alternating magnetic field is applied. IONPs are commonly synthesized by the coprecipitation of aqueous iron salts in the presence of a strong base [16-18]. The biocompatibility of this platform has been demonstrated in vitro and in vivo. Currently, dextran-coated IONPs have been approved by the FDA as MRI contrast agents for liver imaging $[19,20]$. These properties have allowed the use of IONPs for different applications such as vectors for magnetic drug targeting $[21,22]$ and hyperthermia $(\mathrm{MFH})[10,16,23,24]$ to overcome some of the main challenges in cancer therapy.

2.1. Reducing Severe Side Effects. The use of IONPs for magnetic drug targeting (MDT) was first proposed by Frei [25]. It involves the coupling of anticancer agents to iron oxide nanoparticles, injection of the nanovehicle into the blood stream and its guidance to the tumor area using an externally applied magnetic field (Figure 1). This approach allows higher doses of antineoplastic agents in the tumor region with a reduced overall dose [26]. Thus far, there are numerous in vivo and several clinical trials demonstrating the effectiveness of this technique and its ability to reduce side effects [27, 28].

One of the first in vivo studies that reported the utilization of MDT for cancer treatment was conducted by Widder et al. and involved the use of an iron oxide based nanocarrier [29]. IONPs and adriamycin were entrapped in albumin microspheres that were used to increase the amount of chemotherapeutic agent in the tumor using an external magnetic field. A significant accumulation of adriamycin was observed in the tumor and it was similar to that achieved by administrating a 100-fold higher dose of the free drug. After these results, several preclinical studies involving the use of MDT to reduce the severe side effects induced by anticancer drugs have been reported $[26,30]$.

The antineoplastic drug mitoxantrone produces significant side effects such as heart problems, bone marrow suppression, and paralysis [31]. Several studies have evaluated the effectiveness of MDT to overcome these detrimental side

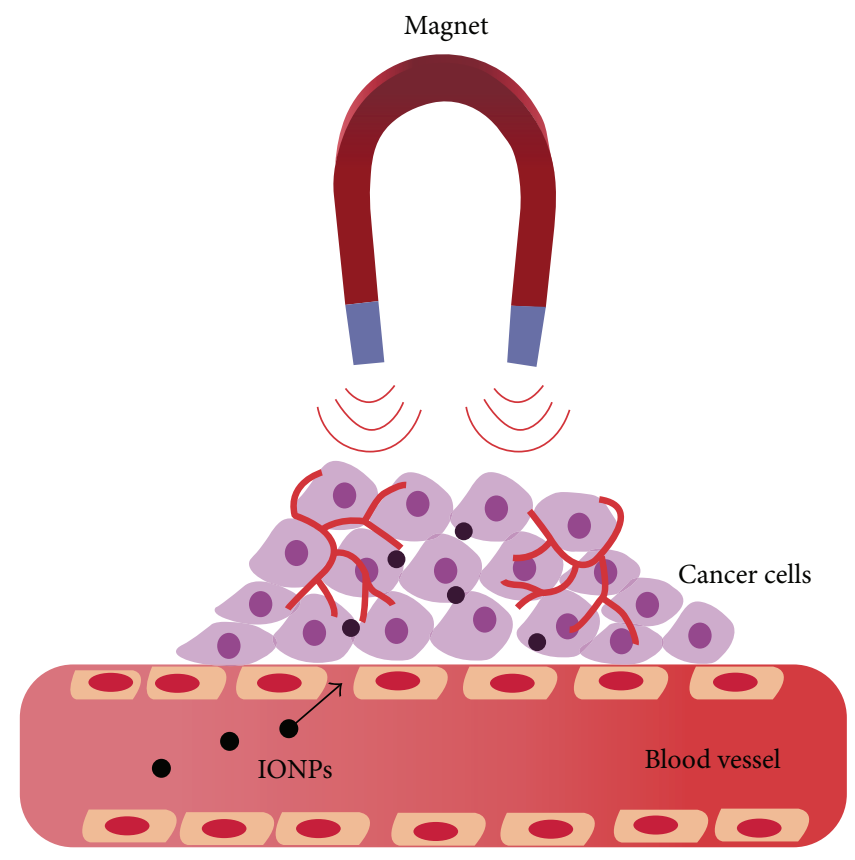

FIGURE 1: Schematic representation of magnetic drug targeting (MDT).

effects. For example, Alexiou et al. used iron oxide based nanoparticles containing mitoxantrone to treat squamous cell carcinoma in rabbits [32]. After intra-arterial injection of this nanocarrier, an external magnetic field was applied on the tumor. The authors reported complete tumor remission after MDT application with minimum toxicity. Later, the same group demonstrated that particles can not only penetrate the vascular wall and concentrate into the tumor, but also can be taken up by the tumor cells, reaching the intracellular space [33]. The therapeutic agent was accumulated in the tumor 50 times higher using MDT than using systemic administered chemotherapy.

More recently, an iron oxide carrier-system containing mitoxantrone was developed and tested in tumors implanted in the hind limbs of rabbits [34]. Results indicated that MDT was able to concentrate the anticancer drug in the tumors at a higher extent (57.2\%) than using the systemic administered chemotherapy $(0.7 \%)$. In addition, this technique induced complete tumor remission and a survival probability of $26.7 \%$.

For decades paclitaxel has been regarded as a successful antineoplastic drug for treating many types of cancer such as breast, ovarian, lung, bladder, and prostate [35]. However, the vehicle used to administer the drug (Cremophor EL/ethanol) induces allergic reactions and histamine release. To overcome this problem, the utilization of magnetoliposomes for MDT has been proposed and evaluated. Zhang et al. synthesized lyophilized paclitaxel magnetoliposomes to treat breast carcinoma [36]. After intravenous (iv) injection of the paclitaxelloaded magnetoliposomes, a magnetic field induced from a permanent magnet was used to lead and accumulated them in the tumor area. The results in this work showed that this platform effectively reached the tumor region and was able 
to induce significant antineoplastic activity, causing lower systemic toxicity than other formulations. Another study evaluated the magnetic vectorization of ultra magnetoliposomes (UML) toward tumors in mice [37]. The findings of this study demonstrated that UML were efficiently internalized by tumor cells when a magnetic field was applied.

MDT has also been explored to target tumors localized in areas of restricted access such as brain tumors. Targeting brain tumors is challenging due to the blood brain barrier that the anticancer drugs need to overcome before reaching the tumor sites. These hurdles make the prognosis of patients with this type of cancer discouraging [38]. Some studies have reported the efficacy of MDT to enhance the delivery of chemotherapeutics to brain tumors with promising results $[39,40]$. The development of iron oxide based nanoparticles encapsulated within the polymer poly[aniline-co- $\mathrm{N}$-(1-onebutyric acid)] aniline (SPAnH) was used to load the anticancer drug epirubicin [39]. This nanovehicle was targeted to the brain combining focused ultrasound and magnetic targeting. The in vivo results confirmed that this synergistic therapy increased the local drug concentration. Similarly, Chertok et al. were able to deliver polyethyleneimine- (PEI) modified magnetic nanoparticles (GPEI) to brain tumors using MDT [40]. While the absence of a magnetic field did not show a significant accumulation of the nanocarrier in the tumor, when the particles were guided by a magnetic field a significant local increase of GPEI was observed.

Although many preclinical studies regarding MDT have been reported, only some clinical phase I/II trials have been conducted. In 1996 the first clinical trial in patients with advanced cancer or sarcomas was carried out [41]. Epirubicin was chemisorbed on IONPs that were covered with anhydroglucose polymers. The results showed that patients treated with MDT exhibited tolerance to this therapy and that the concentration of epidoxorubicin in blood plasma was lower for those patients treated with magnetic drug targeting than patients who received conventional systemic epidoxorubicin.

Later, in 2002 a pharmaceutical company designed and tested magnetic targeted carriers containing doxorubicin for the treatment of liver cancer patients [42]. The use of this iron based nanocarrier for MDT caused the release of the anticancer agent at the tumor site without significant side effects. Despite these promising results, the company did not continue enrolling patients after a phase III clinical trial. In 2004, Wilson et al. reported a clinical study where patients with inoperable hepatocellular carcinoma were treated with a magnetic carrier containing doxorubicin [43]. This study demonstrated that MDT provided selective doxorubicin delivery to the tumor, with a final fraction of treated tumor volume of 0.64 to 0.91 .

Despite of the encouraging results that have been reported about MDT, to date it has not reached the clinical market. Considering that the guidance of the nanoparticles using an external magnetic field is only applicable to superficial tumors, MDT could have tremendous potential for the treatment of these types of tumors. Although there are still some challenges such as optimizing magnetic nanocarriers and magnet design that need to be addressed before translating this technology to a clinically available treatment, we

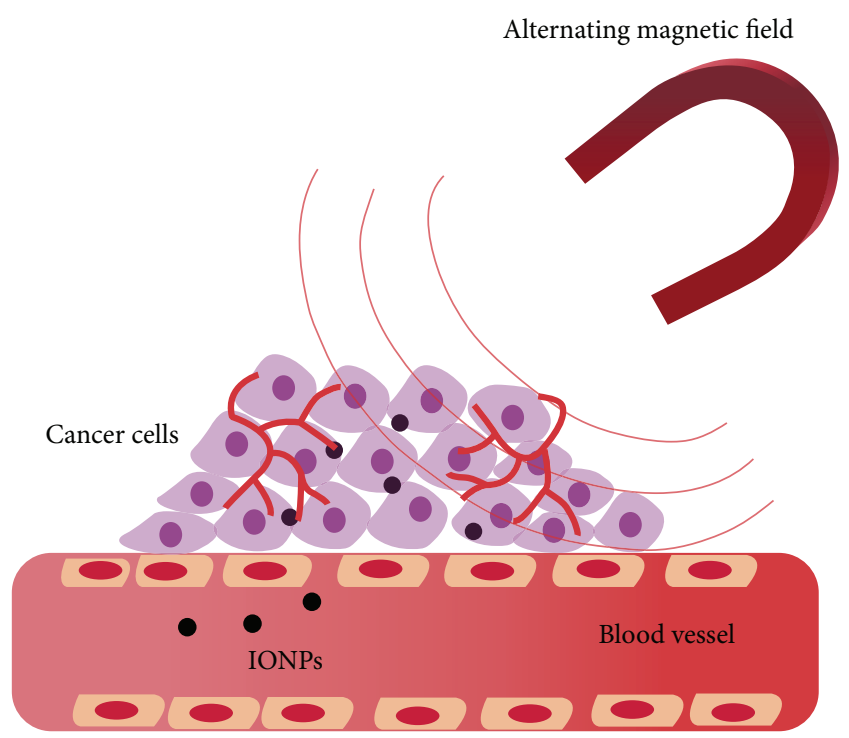

FIGURE 2: Schematic representation of MFH application.

believe that multidisciplinary research will help to overcome these challenges in the future.

2.2. Overcoming Multidrug Resistance. Although the mechanism of cancer multidrug resistance is complex, it has been related to low intracellular accumulation of chemotherapeutics and the ability of cancer cells to repair DNA damage [10]. The use of hyperthermia as an adjunct to improve the therapeutic window of drugs that has shown clinical limitations because MDR has gained great interest. The mechanisms responsible for the enhanced drug cytotoxicity associated with hyperthermia are not yet fully understood but generally involve improved intracellular uptake of drugs due to increased cell membrane permeability and inhibition of DNA repair [44].

Current clinical applications of hyperthermia include surface applicators (RF, microwave, or ultrasound), microwave techniques (small antennas microwave, hot water tubes, and laser fibers), and thermal conduction using thermal chambers [45]. However, these techniques produce significant side effects such as tachycardia, malaise, and impact on tissue metabolism, blood flow, organ function, and tissue repair. For this reason, the use of IONPs to induce local hyperthermia (magnetic fluid hyperthermia (MFH)) without causing damage to adjacent tissues is an attractive approach. It takes advantage of the ability of IONPs to dissipate the magnetic field energy into heat in the presence of an alternating magnetic field (Figure 2). Numerous in vitro and in vivo studies have reported promising results indicating that this practice can promote thermochemosensitization of chemotherapeutic agents $[10,46]$.

Cisplatin is used to treat many types of cancer such as testicular, ovarian, bladder, and non-small cell lung cancer [47]. Nevertheless, its clinical application is limited because some types of malignances do not respond to its therapeutic effect. The thermopotentiation of cisplatin using MFH has widely been studied. One of the first studies 
that demonstrated the enhanced potentiation of cisplatin with IONPs was conducted by Babincová et al. [48]. After these encouraging results, several studies have been reported regarding the combination of cisplatin and $\mathrm{MFH}$ [49-51]. For example, Taylor et al. used carbon-encapsulated iron oxide nanoparticles to treat DU-145 cells using cisplatin and $\mathrm{MFH}$ [50]. The results showed that this combined treatment was more effective than each separate treatment. Similarly, Petryk et al. demonstrated that cisplatin in combination with $\mathrm{MFH}$ was able to induce higher toxic effects than the treatments alone in a murine mammary adenocarcinoma model [52]. Lee et al. investigated the in vitro thermochemosensitization of cisplatin using MFH [51]. In this study, the treatment sequence was evaluated in Caco-2 cancer cells and the results were compared to hyperthermia induced by hot water. For all treatment sequences used, $\mathrm{MFH}$ was more effective in enhancing the cytotoxicity of cisplatin than hot water hyperthermia at the same conditions. The highest toxicity was obtained when cisplatin and $\mathrm{MFH}$ were applied at the same time. Later, Alvarez-Berríos et al. demonstrated that one of the mechanisms by which MFH potentiates cisplatin toxicity is an increase in membrane fluidity [18]. The heat released from IONPs was able to improve drug cytotoxicity by increasing the intracellular accumulation of cisplatin through increased membrane fluidity.

Other anticancer agents have also been explored with this technique. For example, Brusentsov et al. demonstrated that by combining a dextran ferrite- and citrate-coated magnetic nanoparticles with melphalan or cyclophosphamide the lifespan of mice increased by $180 \%$ and $290 \%$, respectively, with significant tumor remission [53]. The in vitro and in vivo thermopotentiation of geldanamycin, an HSP 90 inhibitor using IONPs was evaluated in a B16 melanoma model [54]. Complete tumor remission in five out of nine animals was observed after application of the combined treatment. Similarly, the thermochemosensitization of 5-bromotetrandrine (5-BrTet) and daunorubicin using MFH was observed in an in vivo model of leukemia [55]. The results indicated that tumor size reduction was evident when the combined treatment was applied. Recently, Ren et al. studied the in vivo thermopotentiation of adriamycin using multidrug resistance protein inhibitor-conjugated IONPs [56]. A significant decrease in tumor size was observed in those animals that were exposed to $\mathrm{MFH}$ in combination with the anticancer drug. On the other hand mice that were not exposed to this combined treatment showed an increase in the tumor size.

The enhanced potentiation of bortezomib with $\mathrm{MFH}$ has also been investigated [16, 17]. Bortezomib is a proteasome inhibitor that has been approved by the FDA for multiple myeloma, but its clinical use is limited due to clinical toxicity and drug resistance [57, 58]. Alvarez-Berríos et al. treated bortezomib resistant and non-resistant cancer cells with bortezomib and $\mathrm{MFH}$ [16]. This combined treatment was effective in reducing cell viability in both resistant and non-resistant cancer cells. These results revealed that the utilization of IONPs as nanoheaters to induce localized hyperthermia can sensitize otherwise multidrug-resistant cells. In addition, this approach can not only overcome multidrug resistance, but also help to decrease side effects.
Although several studies evaluating the effectiveness of $\mathrm{MFH}$ in humans have been reported [59, 60], to date no clinical application of $\mathrm{MFH}$ in combination with anticancer drugs has been reported. There are still some challenges with this technology such as improving IONPs concentration and heat generation in the tumor that need to be addressed before it is translated to the clinic. However, based on the encouraging results obtained we anticipate that in the future this approach will gain significant progress to reach the clinical market.

2.3. Fighting Metastasis. The decisions for the treatment of metastatic disease highly depend on diagnostic tools that have the capability of detecting the exact place where metastatic cancer cells are housed [61]. The imaging technology used currently in clinical settings is not precise on finding singlet cells hidden in other organs beyond the primary tumor [62]. The use of IONPs as contrast imaging agents seems to be a powerful tool for metastases diagnosis. IONPs can be modified with molecules that target specific surface receptors or antigens on metastatic cancer cells, allowing the tracking of targeted cells using MRI (Figure 3). The detection of lymph node metastases using IONPs has been explored in the last years, showing promising results $[63,64]$. Harisinghani et al. conducted a study to prove if highly lymphotropic superparamagnetic particles could be used simultaneously with high resolution MRI in order to expose small nodal metastases [65]. This study enrolled eighty patients with T1, T2, or T3 prostate cancer. Each patient was tested by MRI before and after 24 hours of intravenous injection of $2.6 \mathrm{mg}$ of iron per $\mathrm{kg}$ of body weight of the lymphotropic superparamagnetic particles. The high resolution MRI in conjunction with the magnetic nanoparticles allowed a node by node analysis and the detection of small lymph node metastases in patients with prostate cancer. The results obtained from a meta-analysis established that enhanced MRI (superparamagnetic iron oxide nanoparticles (SPIONPs) in combination with MRI) is a sensitive and specific approach for lymph node metastasis detection [63]. While SPIONPs-enhanced MRI had an overall sensitivity of 0.88 and specificity of 0.96 , conventional MRI had a sensitivity of 0.63 and specificity of 0.93 . Similarly, a more recent meta-analysis demonstrated that SPIONPs-enhanced MRI offers higher diagnostic performance, showing better sensitivity (0.90) and specificity (0.96) for the detection of lymph node metastasis than conventional MRI (sensitivity of 0.39 and specificity of 0.90 ) [66].

SPIONPs-enhanced MRI has also been studied for the detection of other cancer metastases. For example, Leuschner et al. studied the ability of targeted SPIONPs to accumulate in metastatic breast cancer cells and to improve the sensitivity of MRI [67]. The surface of the nanoparticles was modified with luteinizing hormone releasing hormone (LHRH) and tested in breast cancer cell lines and in animal models. Nanoparticles were poorly internalized by macrophages and highly incorporated into metastatic cancer cells present in the lungs. Authors claimed that the use of these targeted nanoparticles may serve as a contrast agent for MRI imaging, increasing the sensitivity for the detection of metastasic cancer cells in lymph nodes, bones, and peripheral organs. 


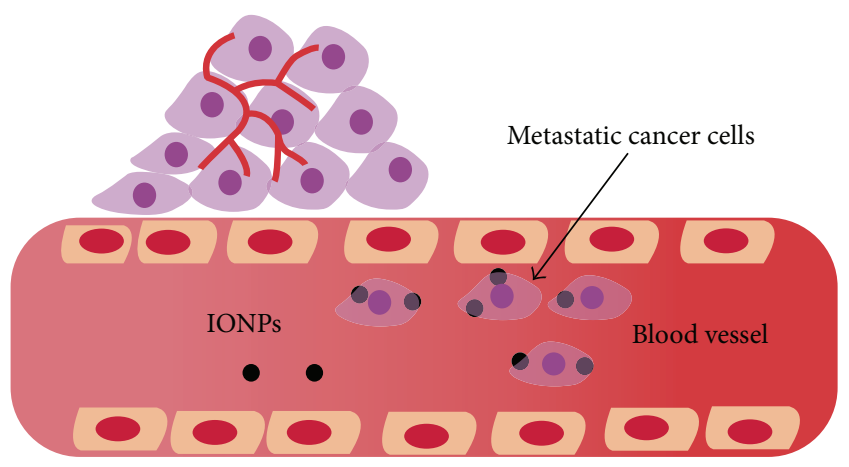

FIGURE 3: Schematic representation of the capability of IONPs for detecting metastatic cancer cells.

Similarly, Vu-Quang et al. reported a targeted IONPs system for the diagnosis of liver metastasis, which occurs in patients with colon, breast, lung, and gastric cancer [68]. IONPs were functionalized with a group of glucose polymers named betaglucan and their ability to target liver and lymph nodes in a metastatic mouse liver model was studied using MRI. The utilization of glucan coated nanoparticles revealed macro and micro metastasized regions in the liver.

A more recent study, reported the utilization of IONPs covered with folic acid to target metastatic ovarian cancer cells present in the whole blood using a magnetic field [69]. The study was able to detect metastatic ovarian cancer cells from the blood of five out of ten patients' whole blood. Although metastasis is still a prevalent challenge in cancer, the use of IONPs for its early diagnosis seems to have a bright future. Since the surface of IONPs can be decorated with different molecules, IONPs have tremendous potential, not only for the diagnosis of metastatic cancer cells, but also for the treatment of metastasis.

\section{Mesoporous Silica Nanoparticles}

Mesoporous materials, which are defined according to IUPAC as materials containing pores with diameters between 2 and $50 \mathrm{~nm}$, have attracted significant attention for their potential use in the biomedical field. Among these inorganicbased materials, MSNs have become the most investigated material in the areas of nanomedicine and biotechnology. MSNs are solid materials, which contain hundreds of empty channels (mesopores) arranged in a 2D network of honeycomb-like porous structure. This ordered pore network, with its size homogeneity, allows for high loading capacity and controlled release kinetics. MSNs are synthesized following a surfactant-templated synthetic approach using tetraethylorthosilicate (TEOS) as silica precursor for the condensation reaction under either basic or acidic conditions. After the material has been synthesized, the surfactant is removed by calcination or acid wash to afford MSNs [70].

As a potential alternative to the current treatments, MSNs have several unique features such as high specific surface area, large pore volume, tunable pore structures, high biocompatibility, and chemical stability. Moreover, MSNs are physically customizable, nontoxic, and safely carry anticancer agents

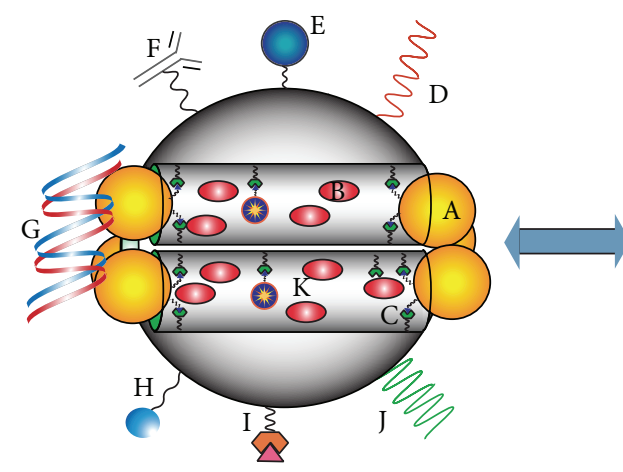

(a)

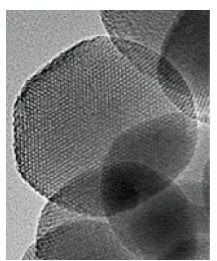

(b)
FIGURE 4: Schematic representation of MSNs as a platform for drug delivery (a). (A) Nanoparticles attached to MSNs as functional gatekeepers. (B) Hydrophobic/hydrophilic guest molecules entrapped in the interior of the nanochannels. (C) Stimuli-responsive linkers, which chemically attach MSNs and gatekeepers. (D) Grafting with a protecting polymer, such as PEG, shields the MSN surface from interacting with opsonizing proteins. (E) Bioimaging agents such as magnetic nanoparticles, quantum dots, or fluorophores. (F) Targeting ligands such as antibodies. (G) Complexation with plasmid DNA. (H) Additional ligands such as cell penetrating peptides. (I) Incorporation of a diagnostic label. (J) Stimuli-responsive polymers. (K) Attachment of functional groups that could modify the metabolism of cells. Transmission electron microscopy image of MSNs (b) [79].

in vivo $[14,71]$. Because of these structural properties the drug loading capacity of either hydrophobic or hydrophilic drugs is increased, making MSNs a promising drug delivery carrier for cancer therapy [72-74]. Additionally, MSNs can be selectively functionalized in both the interior (mesoporous channels) and exterior surfaces with a wide variety of functional groups including small molecules, polymers, and biomolecules [70,75]. The chemical flexibility of this silica-based platform and the vast possibilities of surface functionalization have allowed researchers to develop drug delivery systems with target-specific, stimuli-responsive, and biodegradable properties (Figure 4). Furthermore, a vast variety of contrast agents can be readily incorporated to MSNs. The modification of MSNs with imaging agents for techniques such as optical, MRI, CT, and PET has already been reported in the literature $[76,77]$. The combination of the capabilities of MSNs as therapeutic and diagnostic platform has resulted in theranostic systems. The advantages and efficacy of MSN-based theranostic carriers has also been demonstrated in in vitro and in vivo settings [78]. In addition, MSNs are also an excellent system to develop combinational therapy approaches for cancer treatment.

The use of MSNs for the delivery of anticancer drugs to enhance the therapeutic efficacy against cancer has made significant progress in the last decade. The ability of MSN platform to safely carry and deliver anticancer therapeutics in a target-specific, stimuli-responsive control fashion has been demonstrated in vitro and in vivo with a wide variety of cancers. Nevertheless, in recent years, a great deal of attention has been paid to the use of MSN systems for the 
enhancement of chemotherapy efficacy and inhibitions of multidrug resistance (MDR) and cancer cell metastases.

\subsection{Reducing Severe Side Effects. Conventional chemotherapy} involves the use of agents that are toxic and lack the ability to discriminate between normal and cancerous cells [8083]. For this reason, reducing the side effects induced by chemotherapy is critical to make this therapy more efficient, safer, and patient-friendly. Several approaches have been proposed to address these issues, such as increasing the amount of anticancer drugs loaded to nanoparticles and control their release by stimuli-responsive strategies. In addition, optimizing the intracellular delivery of therapeutic drugs by using nanocarriers as organelle specific delivery systems is a promising alternative to enhance the therapeutic efficacy of antineoplastic agents. Targeted delivery and responsive release of therapeutic drugs based on the difference between tumor tissue/cells and normal ones are a strategy that has also been successfully demonstrated. Because of their outstanding structural properties MSNs are an excellent platform to enhance the target-specificity, safety, and therapeutic efficacy of anticancer drugs. There are many studies that have evaluated their efficacy in different types of malignances with optimistic results. For example, pancreatic cancer is the fourth leading cause of cancer death in the United States. This is one of the most difficult conditions to treat, with a 5-year survival at about 6\% [84]. The horrific prognosis of pancreatic cancer is a result of late diagnosis and the tumor's refractory behavior toward current drug treatment. Gemcitabine is the standard chemotherapeutic drug for this type of cancer, providing a median survival of 5.65 months. Combinations of gemcitabine with other chemotherapeutic drugs and targeted therapies have so far failed to provide any significant improvement [85]. Gurka et al. studied a urokinase-type plasminogen activator receptor (uPAR) targeted, $\mathrm{pH}$-responsive MSN system loaded with gemcitabine and indocyanine green (ICG) as a theranostic MSN-based system for the treatment of pancreatic cancer [86]. UPAR is overexpressed in pancreatic cancer cells and ICG is a multispectral optoacoustic tomography (MSOT) contrast agent that has been used for both in vitro and in vivo assays. The results of this study demonstrated an increase in the specificity of the nanoparticle for pancreatic cancer cells resulting in improved diagnostic accuracy and drug delivery while sparing normal tissues. On the other hand, fluorescence microscopy showed minimal fluorescence for untagged MSNs, while significant fluorescence was observed for uPA targeted MSNs. Additionally, pretreatment using UPAR blocking antibody caused minimal fluorescence confirming that the fluorescence of the uPA targeted MSNs was attributable to UPAR binding. Biodistribution studies performed on S2VP10 mice showed greatest accumulation within the spleen for the untargeted MSNs, whereas substantial binding of uPA targeted MSNs particles within the orthotopic S2VP10 mouse tumor was observed with reduced secondary binding in the specimen's liver, spleen, and kidney. These findings illustrated that uPA targeted, $\mathrm{pH}$-responsive MSN delivery system can be a promising strategy for the treatment of pancreatic cancer with minimal side effects.
Bladder cancer is among the five most common cancers. This year 76,960 new cases of bladder cancer are expected and 16,390 deaths are estimated to be caused by this type of cancer [1]. Nearly $50 \%$ of all bladder cancer patients are diagnosed while the tumor is in situ; for this reason the most widely practiced treatment is a surgical procedure known as transurethral resection of bladder tumor (TURBT). However, due to the high rate of recurrence among bladder cancer patients (50-90\%), it is necessary to use other types of treatments in order to assure complete elimination of cancer. This has been achieved to a large extent by treating patients with intravesical chemotherapy, where the drug is injected intravenously and/or combining it with TURBT. However, this therapy is limited due to the rapid clearance of the drugs from the bladder and low exposure of the tumor site to the chemotherapeutic agents, which can be overpowered by employing nanocarriers for delivering the drug. Taking advantages of the capabilities of MSNs, Zhang et al. reported a pH-responsive, thiol (-SH) functionalized MSNs (MSNPs-Cyclodextrin- $\left.\left(\mathrm{NH}_{2}\right)-\mathrm{SH}\right)$, encapsulating doxorubicin (DOX) for the treatment of bladder cancer [87]. The authors suggested that the introduction of thiol groups, in the surface of the material, would increase the adhesion of MSNs to the mucous membrane of the urothelium through the formation of disulfide bonds. This will allow the sustained release of the loaded anticancer drugs from MSNs during intravesical chemotherapy. In vitro experiments showed that significant fluorescence was observed on the bladder walls for fluorescein labelled MSNs-CD- $\left(\mathrm{NH}_{2}\right)-\mathrm{SH}$, while a material lacking thiol groups (MSNPs-CD-NH ${ }_{2}$ ) showed much lower fluorescence. These results confirmed that the presence of thiol groups on the surface of MSNs enhanced the mucoadhesive capacity for the mucous membrane of the urothelium due to its ability to form covalent bonds between its thiol groups and the glycoproteins in mucin protein. DOX was efficiently loaded into the mesopores of MSNs-CD- $\left(\mathrm{NH}_{2}\right)-\mathrm{SH}$, and its release was triggered by acidic pH conditions. Dox@MSNPs$\mathrm{CD}-\left(\mathrm{NH}_{2}\right)-\mathrm{SH}$ can induce cytotoxicity against UMUC3 bladder cancer cells with an $\mathrm{IC}_{50}$ of $3.92 \pm 1.06 \mu \mathrm{g}$ Dox $\mathrm{mL}^{-1}$. This report thereby demonstrated promising results with functionalized MSNs for mucoadhesive and sustained drug delivery system for superficial bladder cancer therapy.

One of the major issues of anticancer drugs is that despite their advantageous pharmacological activities, most of them cannot be translated into clinical application due to their poor solubility, which usually leads to poor bioavailability. Recently, Jambhrunkar et al. showed a scheme to encapsulate curcumin in the pores of MSNs (MCM-41) through a hydrogen-bonding approach [88]. This strategy improved aqueous solubility, drug release, and cellular delivery of curcumin. These advantageous pharmacological properties resulted in significantly higher cell cytotoxicity toward SCC25, a skin cancer cell line (Figure 5). Celastrol is a traditional herbal medicine known for its anti-inflammatory and anticancer activities. When the apoptotic effects induced by celastrol-loaded MSNs was compared to the same concentrations of free celastrol, a considerably higher efficacy was found when celastrol was delivered by the MSN platform [89]. 


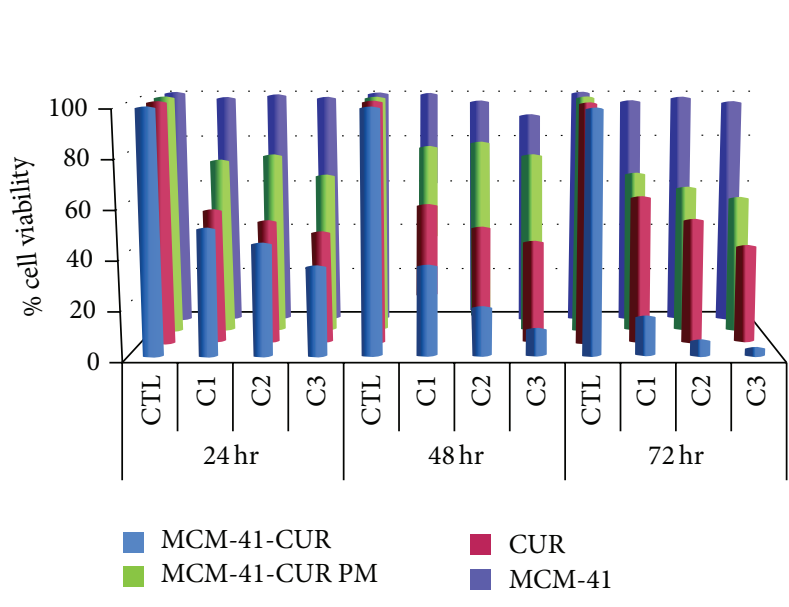

(a)
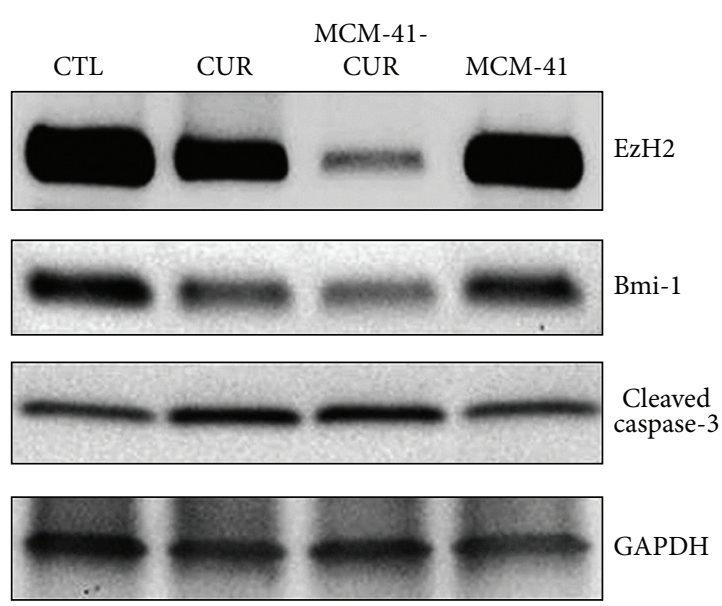

(b)

FIGURE 5: In vitro cytotoxicity of pure curcumin, MCM-41-CUR and MCM-41-CUR PM in SCC-25 cell line after 24, 48, and 72 h (a). CTL, $\mathrm{C} 1, \mathrm{C} 2$, and C3 denote the control group, curcumin equivalent dose of $15 \mathrm{ppm}, 30 \mathrm{ppm}$, and $45 \mathrm{ppm}$, respectively, used in the cell cytotoxicity experiment. Protein expression studies using Western blot analysis for pure curcumin, MCM-41-CUR and MCM-41 (b) [88].

Other groups have also explored the use of aptamerfunctionalized MSNs loaded with DOX for the treatment of colon cancer [13]. Combinational therapies have also been investigated using MSNs. Wang et al. have demonstrated synergistic effect between chemotherapy and photothermal therapy using a drug-loaded MSN platform (MSCN-PEGHB5/DOX) that led to significant cytotoxicity to HER2positive breast cancer cells [90]. Theranostic alternatives in cancer therapy have attracted significant attention in recent years. Lanthanide doped MSNs have been successfully used as theranostic system to integrate dual-imaging, targeting, and therapeutic treatment in multifunctional diagnosis drug delivery systems [91-93].

3.2. Overcoming Multidrug Resistance. Several types of cancer have the ability to develop MDR to traditional therapies. The growing prevalence of MDR needs in-depth research and the development of novel approaches for its treatment. MDR has been one of the biggest impediments to the success of cancer chemotherapy that results when diseases become tolerant to pharmaceutical treatments. Although many types of cancers are initially susceptible to chemotherapy, over time they can develop resistance through a handful of mechanisms. The most generic reason for MDR has been conferred to the presence of at least two molecular "pumps" in tumor cell membranes that actively expel chemotherapy drugs from the interior. P-glycoprotein and the multidrug resistanceassociated protein (MRP), belonging to the ATP-binding cassette family, are the efflux pumps that render the cells resistant to anticancer drugs by altering drug accumulation within the cells. Other mechanisms operating at cellular levels that contribute to MDR include (1) the acquired resistance altered target enzyme (e.g., mutated topoisomerase II); (2) the increased drug degradation due to altered expression of drug-metabolizing enzymes; (3) the drug inactivation due to conjugation with increased amounts of glutathione and the enhanced DNA repair; and (4) the failure to undergo apoptosis as a result of mutated cell cycle proteins such as p53 $[94,95]$.

The interest of developing nanoparticle-based drug delivery systems to reverse MDR in cancer has been growing in recent years [96-98]. For example, it has been reported that nanoparticles can bypass the efflux pump transport and accumulate in cells by passive or active targeting $[99,100]$. Thus, anticancer agents can be efficiently delivered by nanoparticles into MDR cells to reach intracellular therapeutic drug levels to kill cancer cells $[101,102]$.

P-glycoprotein (P-gp) is the best clinically studied drug efflux pump [12]. P-gp mediates resistance to a broad spectrum of anticancer drugs such as DOX, daunorubicin, epirubicin, and paclitaxel. These agents are commonly used in the treatment of breast cancer [103]. The combinatorial approach of chemotherapy and gene therapy has proven to be an effective pharmacologic strategy in overcoming MDR $[104,105]$. Recently, Wang et al. took advantage that CD44 antigen is overexpressed in breast cancer stem cells and MDR breast cancer cells [106-108]. The data showed that CD44abmodified MSNs increased cytotoxicity and enhanced the downregulation of P-glycoprotein in comparison to CD44 antibody [109]. Flow cytometry assay results showed increased fluorescence for DOX-loaded MSNs (DMSNs) and CD44ab-modified DMSNs in comparison to free DOX. Compared to the cells treated with free DOX, the resistant cells treated with DMSNs led to increased DOX fluorescence, demonstrating the efficiency of MSNs to bypass the MDR efflux system and increase the accumulation of anticancer drugs in MDR cancer cells. Moreover, DOX fluorescence from cells treated with CD44ab-modified DMSNs was 1.3-fold higher than fluorescence with DMSNs. These results demonstrated that a higher cellular uptake of DOX can be achieved in MCF-7/MDR1 cells by targeting DMSNs with CD44ab. Western blot analysis was used to detect the expression of P-gp in MCF-7/MDR1. The data showed more significant reduction on P-gp expression level for 
CD44ab-modified DMSNs than with CD44ab alone, or the combinations DMSNs + CD44. Thereby, Western blot analysis corroborated the increased cytotoxicity and enhanced reversal of MDR for CD44ab-modified DMSNs. Animal studies of the MDR breast cancer xenografts demonstrated that CD44ab-modified MSN-based drug delivery system remarkably induced apoptosis and inhibited the tumor growth. In addition, the examination of the tumor DOX retention showed higher intratumoral accumulation of DOX in the case of DMSNs than free DOX. Nonetheless, CD44abmodified DMSNs showed even higher DOX distribution (1.7-fold DOX) than that of DMSN material. The evaluation of the therapeutic efficacy of CD44ab-modified DMSNs in vivo, using MCF7/MDR1 xenografts, showed significant shrinkage of tumor with reduction of about $65 \%$ tumor weight compared with control groups. In summary, CD44abmodified MSNs provided active target, which promoted more cellular uptake of DOX in the MDR cells and more retention of DOX in tumor tissues than the nonmodified MSN material. Therefore, CD44ab-modified MSNs demonstrated superior therapeutic efficacy against MDR breast cancer.

Stimuli-responsive MSN-based drug delivery systems can temporally and spatially regulate the drug-releasing pattern to overcome MDR [110-112]. Zhang et al. utilized this approach to formulate a $\mathrm{pH}$-responsive polymer-lipid combined layer coated MSNs (PLS-MSNs) to enhance the antitumor efficacy against MDR cancer cells [113]. The breast cancer resistance protein (BCRP), which is overexpressed in breast cancer, leukemia, pancreatic cancer, and hepatocellular carcinoma patients [114-117], was used as a target biomarker. BCRP is capable of effluxing a broad range of anticancer agents such as mitoxantrone and camptothecin analogs. Pluronic block copolymers are known to be effective inhibitors of P-gp, multidrug resistance protein (MRP), and BCRP. The use of this platform resulted in the chemosensitization effects in MDR tumors [15, 118-120]. The PLS acted as a door blocker that allowed the controlled release of the antitumor drugs after being internalized by cancer cells. Thereby, this strategy not only minimizes toxicity and maximizes the therapeutic effect of the drugs, but also prevents drugs from being pumped out of MDR cancer cells. In this study, PLS-MSN was loaded with CPT-11. Drug release experiments in simulated body fluid showed that PLS-MSNs released more than $85 \%$ of their encapsulated CPT- 11 within $12 \mathrm{~h}$ at $\mathrm{pH}$ 5.0; meanwhile, minimal release of CPT-11 was detected at $\mathrm{pH}$ 7.4. CPT-11-loaded PLS-MSNs resulted in a significantly higher intracellular CPT-11 delivered into MCF7/BCRP cells after $24 \mathrm{~h}$ of incubation in comparison with free CPT-11 (12.9-fold). These results showed a strong anti-MDR effect of PLS-MSNs. Antitumor activity in BALB/c nude mice MCF-7/BCRP drug resistance tumor xenograft model was investigated to clarify the biological roles of PLS-MSN in reversion of MDR. The final results of their study showed an enhanced cellular uptake of PLS-MSNs by tumor cells, thus improving the efficiency of the active agent (CPT-11). In addition, it was demonstrated that PLS-MSNs could block the efflux transporter and inhibit BCPR mediated CPT-11 pump out of the MDR cells. These promising results provide the basis for future preclinical and clinical testing for developing MSN-based MDR cancer treatment.

Other groups have also looked at the effective circumvention of MDR in cancer cells by an active nuclear-targeted drug delivery of DOX loaded to TAT-conjugated MSNs. This strategy facilitated the nuclear-targeted delivery of DOX in comparison with free DOX [121]. In addition, Jia et al. have fabricated multifunctional MSNs for codelivery of paclitaxel and the anti-MDR agent tetrandrine to successfully reverse the resistance against paclitaxel [122]. Stimuli-responsive hybrid lipid-capped MSNs for drug release and overcoming MDR have been suggested to exhibit higher uptake efficiency, cytotoxicity, and increased intracellular accumulation in resistant MCF-7/Adr cells compared with free drug. All these examples show that the versatility and multifunctionalization of MSNs make this material a promising vehicle for intracellular drug release to overcome MDR [123].

3.3. Fighting Metastasis. Metastasis is defined as the ability of tumor cells to spread from local tissues at the primary tumor site to another at distant secondary site. Metastasis is facilitated by four essential steps: detachment, migration, invasion, and adhesion. Tumor metastases are responsible for approximately $90 \%$ of all cancer-related death [124]. Few patients with metastatic cancer are cured by surgical intervention, and other treatment modalities are limited. Across all cancer types, only one in five patients diagnosed with metastatic cancer will survive more than 5 years [61]. There are handful FDA approved antimetastasis drugs, but so far they have been of little significance into translating them for clinical application. This is attributed to the nonspecific toxicities that result from the lack of ability to recognize the biomarker expression differences between metastatic and normal cells [125]. This nonspecific toxicity can be reduced by making use of nanomedicine to optimize drug distribution, efficient tumor targeting, and guided drug delivery with stimuliresponsive drug release. Ideal nanocarriers to take over this task should incorporate drugs and target molecules to release the cargo in a spatial and temporal fashion. Moreover, these nanocarriers should contain imaging agents alongside for constructing individualized nanomedicines. Owing to the advantaged associated with MSNs, this platform has been used to enhance the efficacy of various antimetastatic drugs and genes, such as silibinin, DOX, and siRNA [95, 126, 127].

Current target-specific antimetastatic treatments mainly rely on antivasculature (antiangiogenesis) and matrix metalloproteinase (MMP) inhibitors. MMP-2 and MMP-9 are overexpressed in advanced stages of cancer, whereas they are minimally expressed in healthy tissue [128]. These elevated levels of MMP in the tumor environment are known to augment the metastatic potency of malignant cells and correlate with tumor progression, angiogenesis, or metastasis. van Rijt et al. have utilized the overexpression of MMP in tumoral tissues to develop a MMP-responsive cisplatin- (CP-) loaded MSNs. The system is capped with avidin protein via a short heptapeptide-biotin linker that is specifically cleaved by MMP9 [129]. This strategy renders controlled release of CP from the MSNs in high MMP9-expressing tumor areas. As a control, the authors synthesized a MSN 
material containing a noncleavable heptapeptide $(\mathrm{nc})$ linker. The release studies under simulated physiological conditions validated the enzymatic-specific release behavior of the entrapped active agents (fluorescein and cisplatin) from the MMP-responsive MSN system by recombinant MMP2/9 enzymes. MMP-triggered release was investigated on two human lung cancer cell lines (A549 and H1299). It was demonstrated via gelatin zymography that the cell-secreted concentration of MMP-9 was able to trigger the release of CP from the MMP-responsive MSN material. On the contrary, the control MSNs containing noncleavable linkers (ncMSN-CP) did not induce any cell death in either cell line. The authors implemented this MMP-responsive CP-loaded MSN system for combinatorial therapy using the proteasome inhibitor bortezomib $(\mathrm{Bz})$. The authors found out that the combination of $\mathrm{Bz}$ with nontoxic dosages of $\mathrm{CP}$ augmented the cytotoxicity in presence of MMP-9. A remarkable 5- to 10 -fold increase in cytotoxicity for nontoxic doses of the CP was observed. These findings illustrated the combinatorial delivery of $\mathrm{CP}$ and $\mathrm{Bz}$ via nanoparticles that induced an additive cytotoxic effect and thus allowed for a reduction of drug dosage. The authors tested the MMP-responsive CP-loaded MSN platform in lung tumors using a $3 \mathrm{D}$ ex vivo tissue culture method. They were able to demonstrate MMP9-trigered release of CP from MSN materials, which induced apoptotic cell death only in lung tumor regions of Kras mutant mice without causing toxicity in tumor areas lacking overexpression of MMP-9 or in healthy mice tissue. Interestingly, MMP-9 expression has been reported as a clinical marker for tumor progression and metastasis [130]. It is possible that these overexpressing MMP-9 tumor areas represent metastasis-prone tumor cells. Therefore, MMP9triggered CP delivery most likely target metastatic tumor cell areas, which may effectively reduce tumor invasion and metastasis. In contrast, Kras mutant mice 3D-LTC exposed to comparable doses of free $\mathrm{CP}$ resulted in apoptotic cell death that did not discriminate much between cancer and healthy tissue. RNA-based therapeutics have also been known to provide a promising strategy to treat cancer by shutting down the genes that translate the specific proteins involved in the mechanism of proliferation, invasion, antiapoptosis, drug resistance, and metastasis [131, 132]. Chen et al. reported on the development of magnetic MSN (M-MSN) based siRNA delivery system with fusogenic peptide KALA modification, using vascular endothelial growth factor (VEGF) as the target gene [127]. VEGF is a gene overexpressed and secreted by most tumor cells that stimulate the formation of new blood vessels through proliferation of endothelial cells $[133,134]$. The carrier was constructed by loading siRNAs into the mesopores of M-MSNs, followed by capping with polyethylenimine (PEI) and further PEGylation with KALA-PEG derivative (siRNA@PEI-PEG-KALA-M-MSNs). The CCK-8 assay for this MSN siRNA delivery system showed favorable biocompatibility and biosafety. In vitro siRNA experiments suggested that the siRNA@PEI-PEG-KALA-MMSN material produced more effective VEGF silencing as compared with commercial analog. Moreover, it displayed negligible cytotoxicity at optimal M-MSN concentration of $80 \mu \mathrm{g} / \mathrm{mL}$ containing $150 \mathrm{nM}$ siRNA dose. The effectiveness of this siRNA delivery system was partially attributed to the presence of the fusogenic peptide on the surface of the nanoparticle. KALA not only increased the endosomal escape of the nanoparticles, but also enhanced their cellular uptake by as much as threefold. This was supported by efficient release of siRNAs from the nanocarrier into the cytoplasm based on confocal microscopy experiments. Biodistribution studies in tumor-bearing mice showed the accumulation of nanoparticles in tumor by MRI up to 7 days after injection. As has been reported in the literature, siRNAs delivered in vivo suffer from rapid degradation in the systemic circulation with a half-life of few minutes [135]. However, using the siRNA@PEI-PEG-KALA-M-MSN system, a 10-fold increase in bioavailability of Cy3-siRNA was achieved. Direct observation and weight measurements also proved the effectiveness of siRNA@PEI-PEG-KALA-MMSNs to inhibit the growth of a subdermal tumor xenograft. Further analysis showed that treatment with siRNA@PEIPEG-KALA-M-MSNs effectively knocked down VEGF levels by $\sim 70 \%$ in tumors. The effect in the reduction of microvessel density in the tumor tissue resulted in a remarkable tumor growth inhibition. In order to determine the effectiveness of the MSN delivery system to suppress tumor growth in the metastasized organs, systemic assessment was performed. The analysis of liver treated with siRNA@PEI-PEG-KALAM-MSNs showed negligible liver metastasis; on the contrary, massive tumor occupancy in the livers was found in the untreated control groups. Consistently, siRNA@PEI-PEGKALA-M-MSNs significantly inhibited the tumor nodule formation by metastatic lung cancer cells, at least up to the examination period of 15 days. Overall, this MSN platform is delivered not only to the primary tumor site, but also to the metastatic site.

Metastasis obstructs the seemingly insurmountable goal of ultimate cancer therapy. Moreover, traditional studies on nanotechnology based anticancer therapies have not included metastasis, since the first line demonstration of anticancer efficacy is primary tumor growth inhibition. Thus, the ability to effectively treat cancer is largely dependent on the ability to eliminate and perhaps even reverse the process of metastasis.

\section{Conclusion and Future Perspective}

Among the different nanomaterials that have been developed for the treatment and diagnosis of cancer, mesoporous silica nanoparticles and iron oxide nanoparticles hold a great potential due to their interesting properties such as high payload, response to external magnetic fields, ease of surface engineering to circumvent unfavorable biological interactions, and excellent biocompatibility, thereby giving us a platform to overcome the main challenges in cancer treatment today.

The exclusive properties of IONPs allow their use as nanoheaters, contrast imaging agents, and drug delivery systems in the fight against cancer. Although IONPs have demonstrated to decrease the detrimental side effects induced by conventional chemotherapy, to date MDT has not reached the clinical market. We believe that the interaction of different research areas such as chemistry, biology, and medicine will 


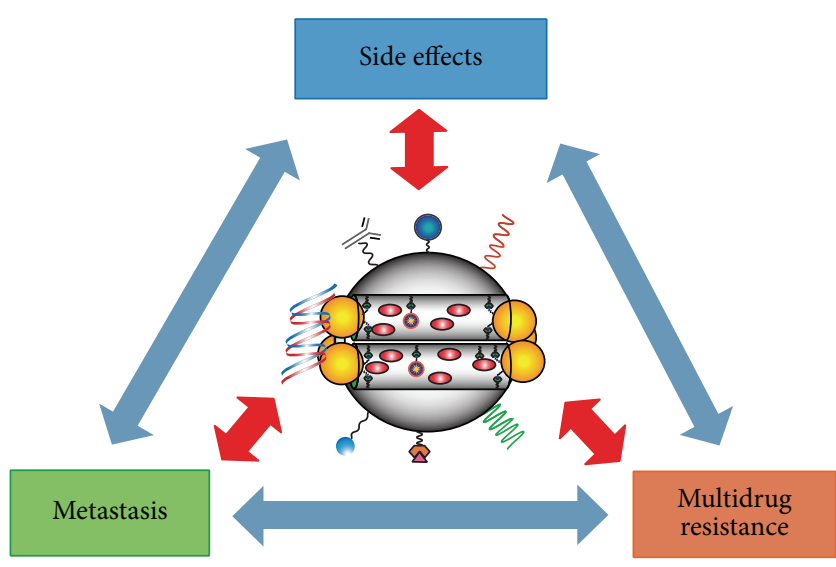

FIGURE 6: Schematic representation of MSN platform and its interaction with the three main obstacles described in this review for cancer treatment.

provide the necessary tools to overcome the major challenges that prevent this technology from being translated to the clinic. Moreover, the enhanced anticancer efficiency induced by MFH provides an alternative platform for reversion of MDR. There are no clinical trials regarding the use of MFH in combination with anticancer drugs; however, this is an active field of research and we envision that in the future this technology will gain significant progress to reach the clinical market. Additionally, the utilization of IONPs as imaging contrast agents has proven to be a promising tool for detection of metastatic cancer cells and we anticipate that their use will have a bright future not only for the diagnosis of metastatic cancer cells, but also for the treatment of metastasis.

On the other hand, MSNs are versatile materials with outstanding structural and chemical properties that can be easily manipulated to design optimal drug delivery platforms. As we have illustrated in this review, in the case of reducing severe side effects, overcoming MDR, and fighting metastasis, MSN-based systems have shown tremendous progress in in vitro and in vivo settings to reduce or eliminate those major issues for cancer treatment. Nevertheless, as far as we know, there are no MSN-based formulations for cancer treatment undergoing clinical trials yet. This situation challenges current researchers in this field to push forward the translation of MSN platform into clinic.

We envision that novel developments for the synthesis and functionalization of MSN materials will enhance the application of this delivery system in biomedicine and also the need to translate this platform from the bench to bedside. From our point of view, some areas that need to be further explored are (1) the development of MSN materials that can be degraded under specific conditions inside the body without producing any toxicity and can be rapidly excreted through the renal excretion pathway; (2) further developments in combination therapy using MSNs as nanocarriers are required, where the therapeutic agents are not only combined anticancer drugs but siRNA, DNA, and therapeutic proteins as well; (3) the fabrication of MSN-based platforms with long circulation time for detection and/or elimination of metastatic cells in blood stream is an area that has not been investigated; (4) in vivo experiments evaluating the performance of MSNs in orthotopic and genetically engineered mouse models have to thoroughly not only explore the efficacy of the MSN formulations in primary tumors, but also evaluate the effect on metastasized secondary tumors. Promising results have been recently reported in the literature for pancreatic cancer using MSNs for the delivery of irinotecan in an orthotopic Kras-derived pancreatic ductal adenocarcinoma model [136].

Multidrug-resistant cancer tissues are closely related to the metastatic process that results in the generation of secondary tumors. These issues create the need for using highly toxic drugs or their combination to treat the tumors, which at the end increase the side effects of the treatment. MSN is a promising drug delivery platform that can have a major impact in each of these issues to improve the efficacy and safety of the treatment (Figure 6).

There is no doubt that MSNs and IONPs can overcome some of the main issues associated with cancer side effects, drug resistance, and metastasis. Nevertheless, more extensive preclinical and clinical testing is necessary for this new and rapidly expanding field to conquer the remaining challenges for moving these platforms toward clinical applications.

\section{Competing Interests}

The authors declare that there are no competing interests regarding the publication of this paper.

\section{References}

[1] Cancer Facts \& Figures 2016, American Cancer Society, Atlanta, Ga, USA, 2016.

[2] E. D. S. Tolentino, B. S. Centurion, L. H. C. Ferreira, A. P. de Souza, J. H. Damante, and I. R. F. Rubira-Bullen, "Oral adverse effects of head and neck radiotherapy: literature review and suggestion of a clinical oral care guideline for irradiated patients," Journal of Applied Oral Science, vol. 19, no. 5, pp. 448-454, 2011.

[3] B. A. Chabner and T. G. Roberts Jr., "Timeline: chemotherapy and the war on cancer," Nature Reviews Cancer, vol. 5, no. 1, pp. 65-72, 2005.

[4] A. Nguyen, L. Ho, and Y. Wan, "Chemotherapy and oncolytic virotherapy: advanced tactics in the war against cancer," Frontiers in Oncology, vol. 4, article 145, 2014.

[5] C. B. Matsen and L. A. Neumayer, "Breast cancer: a review for the general surgeon," JAMA Surgery, vol. 148, no. 10, pp. 971979, 2013.

[6] M. F. Leber and T. Efferth, "Molecular principles of cancer invasion and metastasis (review)," International Journal of Oncology, vol. 34, no. 4, pp. 881-895, 2009.

[7] M. J. Sailor and J.-H. Park, "Hybrid nanoparticles for detection and treatment of cancer," Advanced Materials, vol. 24, no. 28, pp. 3779-3802, 2012.

[8] J. L. Vivero-Escoto and Y.-T. Huang, "Inorganic-organic hybrid nanomaterials for therapeutic and diagnostic imaging applications," International Journal of Molecular Sciences, vol. 12, no. 6, pp. 3888-3927, 2011.

[9] X. Hao, X. Hu, C. Zhang et al., "Hybrid mesoporous silicabased drug carrier nanostructures with improved degradability 
by hydroxyapatite," ACS Nano, vol. 9, no. 10, pp. 9614-9625, 2015.

[10] M. Torres-Lugo and C. Rinaldi, "Thermal potentiation of chemotherapy by magnetic nanoparticles," Nanomedicine, vol. 8, no. 10, pp. 1689-1707, 2013.

[11] V. P. Torchilin, "Passive and active drug targeting: drug delivery to tumors as an example," Handbook of Experimental Pharmacology, no. 197, pp. 3-53, 2010.

[12] I.-P. Huang, S.-P. Sun, S.-H. Cheng et al., "Enhanced chemotherapy of cancer using $\mathrm{pH}$-sensitive mesoporous silica nanoparticles to antagonize P-glycoprotein-mediated drug resistance," Molecular Cancer Therapeutics, vol. 10, no. 5, pp. 761-769, 2011.

[13] X. Xie, F. Li, H. Zhang et al., "EpCAM aptamer-functionalized mesoporous silica nanoparticles for efficient colon cancer celltargeted drug delivery," European Journal of Pharmaceutical Sciences, vol. 83, pp. 28-35, 2016.

[14] Y. Wang, Q. Zhao, N. Han et al., "Mesoporous silica nanoparticles in drug delivery and biomedical applications," Nanomedicine: Nanotechnology, Biology, and Medicine, vol. 11, no. 2, pp. 313-327, 2015.

[15] W. Zhang, Y. Shi, Y. Chen, J. Ye, X. Sha, and X. Fang, "Multifunctional Pluronic P123/F127 mixed polymeric micelles loaded with paclitaxel for the treatment of multidrug resistant tumors," Biomaterials, vol. 32, no. 11, pp. 2894-2906, 2011.

[16] M. P. Alvarez-Berríos, A. Castillo, C. Rinaldi, and M. TorresLugo, "Magnetic fluid hyperthermia enhances cytotoxicity of bortezomib in sensitive and resistant cancer cell lines," International Journal of Nanomedicine, vol. 9, pp. 145-153, 2014.

[17] M. P. Alvarez-Berrios, A. Castillo, F. Merida, J. Mendez, C. Rinaldi, and M. Torres-Lugo, "Enhanced proteotoxic stress: one of the contributors for hyperthermic potentiation of the proteasome inhibitor bortezomib using magnetic nanoparticles," Biomaterials Science, vol. 3, no. 2, pp. 391-400, 2015.

[18] M. P. Alvarez-Berríos, A. Castillo, J. Mendéz, O. Soto, C. Rinaldi, and $\mathrm{M}$. Torres-Lugo, "Hyperthermic potentiation of cisplatin by magnetic nanoparticle heaters is correlated with an increase in cell membrane fluidity," International Journal of Nanomedicine, vol. 8, pp. 1003-1013, 2013.

[19] B. Marincek, "Diagnostic improvement in MRI of gynecological neoplasms," Journal Belge de Radiologie, vol. 79, no. 1, pp. 13-17, 1996.

[20] P. Reimer and T. Balzer, "Ferucarbotran (Resovist): a new clinically approved RES-specific contrast agent for contrastenhanced MRI of the liver: properties, clinical development, and applications," European Radiology, vol. 13, no. 6, pp. 12661276, 2003.

[21] S.-H. Hu, Y.-Y. Chen, T.-C. Liu, T.-H. Tung, D.-M. Liu, and S.-Y. Chen, "Remotely nano-rupturable yolk/shell capsules for magnetically-triggered drug release," Chemical Communications, vol. 47, no. 6, pp. 1776-1778, 2011.

[22] E. Amstad and E. Reimhult, "Nanoparticle actuated hollow drug delivery vehicles," Nanomedicine, vol. 7, no. 1, pp. 145-164, 2012.

[23] R. Hu, S. Ma, H. Li et al., "Effect of magnetic fluid hyperthermia on lung cancer nodules in a murine model," Oncology Letters, vol. 2, no. 6, pp. 1161-1164, 2011.

[24] S. Laurent, S. Dutz, U. O. Häfeli, and M. Mahmoudi, "Magnetic fluid hyperthermia: focus on superparamagnetic iron oxide nanoparticles," Advances in Colloid and Interface Science, vol. 166, no. 1-2, pp. 8-23, 2011.

[25] E. H. Frei, "Magnetism and medicine," Journal of Applied Physics, vol. 40, no. 3, pp. 955-957, 1969.
[26] J. Estelrich, E. Escribano, J. Queralt, and M. A. Busquets, "Iron oxide nanoparticles for magnetically-guided and magneticallyresponsive drug delivery," International Journal of Molecular Sciences, vol. 16, no. 4, pp. 8070-8101, 2015.

[27] A. S. Lübbe, C. Alexiou, and C. Bergemann, "Clinical applications of magnetic drug targeting," Journal of Surgical Research, vol. 95, no. 2, pp. 200-206, 2001.

[28] B. Polyak and G. Friedman, "Magnetic targeting for site-specific drug delivery: applications and clinical potential," Expert Opinion on Drug Delivery, vol. 6, no. 1, pp. 53-70, 2009.

[29] K. J. Widder, A. E. Senyel, and G. D. Scarpelli, "Magnetic microspheres: a model system of site specific drug delivery in vivo," Proceedings of the Society for Experimental Biology and Medicine, vol. 158, no. 2, pp. 141-146, 1978.

[30] S. Lyer, R. Tietze, R. Jurgons et al., "Visualisation of tumour regression after local chemotherapy with magnetic nanoparticles-a pilot study," Anticancer Research, vol. 30, no. 5, pp. 1553-1557, 2010.

[31] X. Thomas, M. Elhamri, Y. Chelghoum et al., "Intensive chemotherapy with mitoxantrone administered as a single injection in patients with high-risk acute myeloid leukemia: results of the EMA 2000 trial," Annals of Hematology, vol. 84, no. 6, pp. 376-382, 2005.

[32] C. Alexiou, W. Arnold, R. J. Klein et al., "Locoregional cancer treatment with magnetic drug targeting," Cancer Research, vol. 60, no. 23, pp. 6641-6648, 2000.

[33] C. Alexiou, R. Jurgons, R. J. Schmid et al., "Magnetic drug targeting-biodistribution of the magnetic carrier and the chemotherapeutic agent mitoxantrone after locoregional cancer treatment," Journal of Drug Targeting, vol. 11, no. 3, pp. 139-149, 2003.

[34] R. Tietze, S. Lyer, S. Dürr et al., "Efficient drug-delivery using magnetic nanoparticles-biodistribution and therapeutic effects in tumour bearing rabbits," Nanomedicine: Nanotechnology, Biology, and Medicine, vol. 9, no. 7, pp. 961-971, 2013.

[35] T. M. Mekhail and M. Markman, "Paclitaxel in cancer therapy," Expert Opinion on Pharmacotherapy, vol. 3, no. 6, pp. 755-766, 2002.

[36] J. Q. Zhang, Z. R. Zhang, H. Yang, Q. Y. Tan, S. R. Qin, and X. L. Qiu, "Lyophilized paclitaxel magnetoliposomes as a potential drug delivery system for breast carcinoma via parenteral administration: in vitro and in vivo studies," Pharmaceutical Research, vol. 22, no. 4, pp. 573-583, 2005.

[37] G. Béalle, R. Di Corato, J. Kolosnjaj-Tabi et al., "Ultra magnetic liposomes for MR imaging, targeting, and hyperthermia," Langmuir, vol. 28, no. 32, pp. 11834-11842, 2012.

[38] D. Y. Joh, L. Sun, M. Stangl et al., "Selective targeting of brain tumors with gold nanoparticle-induced radiosensitization," PLoS ONE, vol. 8, no. 4, Article ID e62425, 2013.

[39] H.-L. Liu, M.-Y. Hua, H.-W. Yang et al., "Magnetic resonance monitoring of focused ultrasound/magnetic nanoparticle targeting delivery of therapeutic agents to the brain," Proceedings of the National Academy of Sciences of the United States of America, vol. 107, no. 34, pp. 15205-15210, 2010.

[40] B. Chertok, A. E. David, and V. C. Yang, "Polyethyleneiminemodified iron oxide nanoparticles for brain tumor drug delivery using magnetic targeting and intra-carotid administration," Biomaterials, vol. 31, no. 24, pp. 6317-6324, 2010.

[41] A. S. Lubbe, C. Bergemann, H. Riess et al., "Clinical experiences with magnetic drug targeting: a phase I study with $4^{\prime}$-epidoxorubicin in 14 patients with advanced solid tumors," Cancer Research, vol. 56, no. 20, pp. 4686-4693, 1996. 
[42] Z. Saiyed, S. Telang, and C. Ramchand, "Application of magnetic techniques in the field of drug discovery and biomedicine," Biomagnetic Research and Technology, vol. 1, no. 1, article 2, 2003.

[43] M. W. Wilson, R. K. Kerlan Jr., N. A. Fidelman et al., "Hepatocellular carcinoma: regional therapy with a magnetic targeted carrier bound to doxorubicin in a dual MR imaging/ conventional angiography suite-initial experience with four patients," Radiology, vol. 230, no. 1, pp. 287-293, 2004.

[44] R. D. Issels, "Hyperthermia adds to chemotherapy," European Journal of Cancer, vol. 44, no. 17, pp. 2546-2554, 2008.

[45] M. H. Falk and R. D. Issels, "Hyperthermia in oncology," International Journal of Hyperthermia, vol. 17, no. 1, pp. 1-18, 2001.

[46] S. Likhitkar and A. K. Bajpai, "Magnetically controlled release of cisplatin from superparamagnetic starch nanoparticles," Carbohydrate Polymers, vol. 87, no. 1, pp. 300-308, 2012.

[47] A. Rajeswaran, A. Trojan, B. Burnand, and M. Giannelli, "Efficacy and side effects of cisplatin- and carboplatin-based doublet chemotherapeutic regimens versus non-platinum-based doublet chemotherapeutic regimens as first line treatment of metastatic non-small cell lung carcinoma: a systematic review of randomized controlled trials," Lung Cancer, vol. 59, no. 1, pp. 1-11, 2008.

[48] M. Babincová, V. Altanerová, C. Altaner, C. Bergemann, and P. Babinec, "In vitro analysis of cisplatin functionalized magnetic nanoparticles in combined cancer chemotherapy and electromagnetic hyperthermia," IEEE Transactions on NanoBioscience, vol. 7, no. 1, pp. 15-19, 2008.

[49] A. J. Wagstaff, S. D. Brown, M. R. Holden et al., "Cisplatin drug delivery using gold-coated iron oxide nanoparticles for enhanced tumour targeting with external magnetic fields," Inorganica Chimica Acta, vol. 393, pp. 328-333, 2012.

[50] A. Taylor, Y. Krupskaya, K. Krämer et al., "Cisplatin-loaded carbon-encapsulated iron nanoparticles and their in vitro effects in magnetic fluid hyperthermia," Carbon, vol. 48, no. 8 , pp. 2327-2334, 2010.

[51] J. S. Lee, H. L. Rodríguez-Luccioni, J. Méndez et al., "Hyperthermia induced by magnetic nanoparticles improves the effectiveness of the anticancer drug cis-diamminedichloroplatinum," Journal of Nanoscience and Nanotechnology, vol. 11, no. 5, pp. 4153-4157, 2011.

[52] A. A. Petryk, A. J. Giustini, R. E. Gottesman, P. A. Kaufman, and P. J. Hoopes, "Magnetic nanoparticle hyperthermia enhancement of cisplatin chemotherapy cancer treatment," International Journal of Hyperthermia, vol. 29, no. 8, pp. 845851, 2013.

[53] N. A. Brusentsov, V. A. Polyanskii, Y. A. Pirogov et al., "Antitumor effects of the combination of magnetohydrodynamic thermochemotherapy and magnetic resonance tomography," Pharmaceutical Chemistry Journal, vol. 44, no. 6, pp. 291-295, 2010.

[54] A. Ito, H. Saito, K. Mitobe et al., "Inhibition of heat shock protein 90 sensitizes melanoma cells to thermosensitive ferromagnetic particle-mediated hyperthermia with low Curie temperature," Cancer Science, vol. 100, no. 3, pp. 558-564, 2009.

[55] E. Amstad, J. Kohlbrecher, E. Müller, T. Schweizer, M. Textor, and E. Reimhult, "Triggered release from liposomes through magnetic actuation of iron oxide nanoparticle containing membranes," Nano Letters, vol. 11, no. 4, pp. 1664-1670, 2011.

[56] Y. Ren, H. Zhang, B. Chen et al., "Multifunctional magnetic $\mathrm{Fe}_{3} \mathrm{O}_{4}$ nanoparticles combined with chemotherapy and hyperthermia to overcome multidrug resistance," International Journal of Nanomedicine, vol. 7, pp. 2261-2269, 2012.
[57] B. Piperdi, Y.-H. Ling, L. Liebes, F. Muggia, and R. PerezSoler, "Bortezomib: understanding the mechanism of action," Molecular Cancer Therapeutics, vol. 10, no. 11, pp. 2029-2030, 2011.

[58] N. Neznanov, A. P. Komarov, L. Neznanova, P. Stanhope-Baker, and A. V. Gudkov, "Proteotoxic stress targeted therapy (PSTT): induction of protein misfolding enhances the antitumor effect of the proteasome inhibitor bortezomib," Oncotarget, vol. 2, no. 3, pp. 209-221, 2011.

[59] M. Johannsen, U. Gneveckow, L. Eckelt et al., "Clinical hyperthermia of prostate cancer using magnetic nanoparticles: presentation of a new interstitial technique," International Journal of Hyperthermia, vol. 21, no. 7, pp. 637-647, 2005.

[60] M. Johannsen, B. Thiesen, P. Wust, and A. Jordan, "Magnetic nanoparticle hyperthermia for prostate cancer," International Journal of Hyperthermia, vol. 26, no. 8, pp. 790-795, 2010.

[61] A. Schroeder, D. A. Heller, M. M. Winslow et al., "Treating metastatic cancer with nanotechnology," Nature Reviews Cancer, vol. 12, no. 1, pp. 39-50, 2012.

[62] O. L. Gobbo, K. Sjaastad, M. W. Radomski, Y. Volkov, and A. Prina-Mello, "Magnetic nanoparticles in cancer theranostics," Theranostics, vol. 5, no. 11, pp. 1249-1263, 2015.

[63] O. Will, S. Purkayastha, C. Chan et al., "Diagnostic precision of nanoparticle-enhanced MRI for lymph-node metastases: a meta-analysis," The Lancet Oncology, vol. 7, no. 1, pp. 52-60, 2006.

[64] T. Harada, N. Tanigawa, M. Matsuki, T. Nohara, and I. Narabayashi, "Evaluation of lymph node metastases of breast cancer using ultrasmall superparamagnetic iron oxide-enhanced magnetic resonance imaging," European Journal of Radiology, vol. 63, no. 3, pp. 401-407, 2007.

[65] M. G. Harisinghani, J. Barentsz, P. F. Hahn et al., "Noninvasive detection of clinically occult lymph-node metastases in prostate cancer," The New England Journal of Medicine, vol. 348, no. 25, pp. 2491-2499, 2003.

[66] L. Wu, Y. Cao, C. Liao, J. Huang, and F. Gao, "Diagnostic performance of USPIO-enhanced MRI for lymph-node metastases in different body regions: a meta-analysis," European Journal of Radiology, vol. 80, no. 2, pp. 582-589, 2011.

[67] C. Leuschner, C. S. S. R. Kumar, W. Hansel, W. Soboyejo, J. Zhou, and J. Hormes, "LHRH-conjugated magnetic iron oxide nanoparticles for detection of breast cancer metastases," Breast Cancer Research and Treatment, vol. 99, no. 2, pp. 163-176, 2006.

[68] H. Vu-Quang, M. Muthiah, H. J. Lee et al., "Immune cellspecific delivery of beta-glucan-coated iron oxide nanoparticles for diagnosing liver metastasis by MR imaging," Carbohydrate Polymers, vol. 87, no. 2, pp. 1159-1168, 2012.

[69] W. Liu, L. Nie, F. Li et al., "Folic acid conjugated magnetic iron oxide nanoparticles for nondestructive separation and detection of ovarian cancer cells from whole blood," Biomaterials Science, vol. 4, no. 1, pp. 159-166, 2016.

[70] I. I. Slowing, J. L. Vivero-Escoto, C.-W. Wu, and V. S.-Y. Lin, "Mesoporous silica nanoparticles as controlled release drug delivery and gene transfection carriers," Advanced Drug Delivery Reviews, vol. 60, no. 11, pp. 1278-1288, 2008.

[71] Y. Kuthati, P.-J. Sung, C.-F. Weng, C.-Y. Mou, and C.-H. Lee, "Functionalization of mesoporous silica nanoparticles for targeting, biocompatibility, combined cancer therapies and theragnosis," Journal of Nanoscience and Nanotechnology, vol. 13, no. 4, pp. 2399-2430, 2013.

[72] Y. Chen, H. Chen, S. Zhang et al., "Multifunctional mesoporous nanoellipsoids for biological bimodal imaging and magnetically 
targeted delivery of anticancer drugs," Advanced Functional Materials, vol. 21, no. 2, pp. 270-278, 2011.

[73] W. Zhao, H. Chen, Y. Li, A. Li, M. Lang, and J. Shi, "Uniform rattle-type hollow magnetic mesoporous spheres as drug delivery carriers and their sustained-release property," Advanced Functional Materials, vol. 18, no. 18, pp. 2780-2788, 2008.

[74] H. Tu, Y. Lin, H. Lin et al., "In vitro studies of functionalized mesoporous silica nanoparticles for photodynamic therapy," Advanced Materials, vol. 21, no. 2, pp. 172-177, 2009.

[75] J. E. Lee, N. Lee, H. Kim et al., "Uniform mesoporous dyedoped silica nanoparticles decorated with multiple magnetite nanocrystals for simultaneous enhanced magnetic resonance imaging, fluorescence imaging, and drug delivery," Journal of the American Chemical Society, vol. 132, no. 2, pp. 552-557, 2010.

[76] C. Caltagirone, A. Bettoschi, A. Garau, and R. Montis, "Silicabased nanoparticles: a versatile tool for the development of efficient imaging agents," Chemical Society Reviews, vol. 44, no. 14, pp. 4645-4671, 2015.

[77] J. L. Vivero-Escoto, R. C. Huxford-Phillips, and W. Lin, "Silicabased nanoprobes for biomedical imaging and theranostic applications," Chemical Society Reviews, vol. 41, no. 7, pp. 26732685, 2012.

[78] N.-T. Chen, S.-H. Cheng, J. S. Souris, C.-T. Chen, C.-Y. Mou, and L.-W. Lo, "Theranostic applications of mesoporous silica nanoparticles and their organic/inorganic hybrids," Journal of Materials Chemistry B, vol. 1, no. 25, pp. 3128-3135, 2013.

[79] J. L. Vivero-Escoto, I. I. Slowing, V. S.-Y. Lin, and B. G. Trewyn, "Mesoporous silica nanoparticles for intracellular controlled drug delivery," Small, vol. 6, no. 18, pp. 1952-1967, 2010.

[80] L. Kelland, "The resurgence of platinum-based cancer chemotherapy," Nature Reviews Cancer, vol. 7, no. 8, pp. 573-584, 2007.

[81] M. Markman, "Toxicities of the platinum antineoplastic agents," Expert Opinion on Drug Safety, vol. 2, no. 6, pp. 597-607, 2003.

[82] J. Reedijk, "New clues for platinum antitumor chemistry: kinetically controlled metal binding to DNA," Proceedings of the National Academy of Sciences of the United States of America, vol. 100, no. 7, pp. 3611-3616, 2003.

[83] P. R. Brock, K. R. Knight, D. R. Freyer et al., "Platinum-induced ototoxicity in children: a consensus review on mechanisms, predisposition, and protection, including a new International Society of Pediatric Oncology Boston ototoxicity scale," Journal of Clinical Oncology, vol. 30, no. 19, pp. 2408-2417, 2012.

[84] N. Bardeesy and R. A. DePinho, "Pancreatic cancer biology and genetics," Nature Reviews Cancer, vol. 2, no. 12, pp. 897-909, 2002.

[85] A. Stathis and M. J. Moore, "Advanced pancreatic carcinoma: current treatment and future challenges," Nature Reviews Clinical Oncology, vol. 7, no. 3, pp. 163-172, 2010.

[86] M. K. Gurka, D. Pender, P. Chuong et al., "Identification of pancreatic tumors in vivo with ligand-targeted, $\mathrm{pH}$ responsive mesoporous silica nanoparticles by multispectral optoacoustic tomography," Journal of Controlled Release, 2016.

[87] Q. Zhang, K. G. Neoh, L. Xu et al., "Functionalized mesoporous silica nanoparticles with mucoadhesive and sustained drug release properties for potential bladder cancer therapy," Langmuir, vol. 30, no. 21, pp. 6151-6161, 2014.

[88] S. Jambhrunkar, S. Karmakar, A. Popat, M. Yu, and C. Yu, "Mesoporous silica nanoparticles enhance the cytotoxicity of curcumin," RSC Advances, vol. 4, no. 2, pp. 709-712, 2014.

[89] E. Niemelä, D. Desai, Y. Nkizinkiko, J. E. Eriksson, and J. M. Rosenholm, "Sugar-decorated mesoporous silica nanoparticles as delivery vehicles for the poorly soluble drug celastrol enables targeted induction of apoptosis in cancer cells," European Journal of Pharmaceutics and Biopharmaceutics, vol. 96, pp. 1121, 2015.

[90] K. Wang, H. Yao, Y. Meng, Y. Wang, X. Yan, and R. Huang, "Specific aptamer-conjugated mesoporous silica-carbon nanoparticles for HER2-targeted chemo-photothermal combined therapy," Acta Biomaterialia, vol. 16, no. 1, pp. 196-205, 2015.

[91] X. Hu, M. Wang, F. Miao, J. Ma, H. Shen, and N. Jia, "Regulation of multifunctional mesoporous core-shell nanoparticles with luminescence and magnetic properties for biomedical applications," Journal of Materials Chemistry B, vol. 2, no. 16, pp. 22652275, 2014.

[92] Y. Chen, K. Ai, J. Liu, G. Sun, Q. Yin, and L. Lu, "Multifunctional envelope-type mesoporous silica nanoparticles for $\mathrm{pH}$ responsive drug delivery and magnetic resonance imaging," Biomaterials, vol. 60, pp. 111-120, 2015.

[93] M.-H. Chan and H.-M. Lin, "Preparation and identification of multifunctional mesoporous silica nanoparticles for in vitro and in vivo dual-mode imaging, theranostics, and targeted tracking," Biomaterials, vol. 46, pp. 149-158, 2015.

[94] K. O. Alfarouk, C.-M. Stock, S. Taylor et al., "Resistance to cancer chemotherapy: failure in drug response from ADME to P-gp," Cancer Cell International, vol. 15, article 71, 2015.

[95] S. Chen, Z. Wang, Y. Huang et al., "Ginseng and anticancer drug combination to improve cancer chemotherapy: a critical review," Evidence-Based Complementary and Alternative Medicine, vol. 2014, Article ID 168940, 13 pages, 2014.

[96] Y. Gao, Y. Chen, X. Ji et al., "Controlled intracellular release of doxorubicin in multidrug-resistant cancer cells by tuning the shell-pore sizes of mesoporous silica nanoparticles," ACS Nano, vol. 5, no. 12, pp. 9788-9798, 2011.

[97] Y. Chen, Q. Yin, X. Ji et al., "Manganese oxide-based multifunctionalized mesoporous silica nanoparticles for $\mathrm{pH}$-responsive MRI, ultrasonography and circumvention of MDR in cancer cells," Biomaterials, vol. 33, no. 29, pp. 7126-7137, 2012.

[98] W. C. Zamboni, V. Torchilin, A. K. Patri et al., "Best practices in cancer nanotechnology: perspective from NCI nanotechnology alliance," Clinical Cancer Research, vol. 18, no. 12, pp. 3229-3241, 2012.

[99] B. Li, H. Xu, Z. Li et al., "Bypassing multidrug resistance in human breast cancer cells with lipid/polymer particle assemblies," International Journal of Nanomedicine, vol. 7, pp. 187-197, 2012.

[100] W. Jiang, B. Y. S. Kim, J. T. Rutka, and W. C. W. Chan, "Nanoparticle-mediated cellular response is size-dependent," Nature Nanotechnology, vol. 3, no. 3, pp. 145-150, 2008.

[101] W. Punfa, S. Yodkeeree, P. Pitchakarn, C. Ampasavate, and P. Limtrakul, "Enhancement of cellular uptake and cytotoxicity of curcumin-loaded PLGA nanoparticles by conjugation with anti-P-glycoprotein in drug resistance cancer cells," Acta Pharmacologica Sinica, vol. 33, no. 6, pp. 823-831, 2012.

[102] A. S. Gonçalves, A. S. Macedo, and E. B. Souto, "Therapeutic nanosystems for oncology nanomedicine," Clinical and Translational Oncology, vol. 14, no. 12, pp. 883-890, 2012.

[103] T. Nabekura, "Overcoming multidrug resistance in human cancer cells by natural compounds," Toxins, vol. 2, no. 6, pp. 1207-1224, 2010.

[104] A. M. Chen, M. Zhang, D. Wei et al., "Co-delivery of doxorubicin and Bcl-2 siRNA by mesoporous silica nanoparticles enhances the efficacy of chemotherapy in multidrug-resistant cancer cells," Small, vol. 5, no. 23, pp. 2673-2677, 2009. 
[105] C. E. Ashley, E. C. Carnes, G. K. Phillips et al., "The targeted delivery of multicomponent cargos to cancer cells by nanoporous particle-supported lipid bilayers," Nature Materials, vol. 10, no. 6, p. 476, 2011, Erratum to document cited in CA155:021668.

[106] A. M. Calcagno, C. D. Salcido, J.-P. Gillet et al., "Prolonged drug selection of breast cancer cells and enrichment of cancer stem cell characteristics," Journal of the National Cancer Institute, vol. 102, no. 21, pp. 1637-1652, 2010.

[107] J. W. Cain, R. S. Hauptschein, J. K. Stewart, T. Bagci, G. G. Sahagian, and D. G. Jay, "Identification of CD44 as a surface biomarker for drug resistance by surface proteome signature technology," Molecular Cancer Research, vol. 9, no. 5, pp. 637647, 2011.

[108] D. Wang, J. Huang, X. Wang et al., "The eradication of breast cancer cells and stem cells by 8-hydroxyquinolineloaded hyaluronan modified mesoporous silica nanoparticlesupported lipid bilayers containing docetaxel," Biomaterials, vol. 34, no. 31, pp. 7662-7673, 2013.

[109] X. Wang, Y. Liu, S. Wang et al., "CD44-engineered mesoporous silica nanoparticles for overcoming multidrug resistance in breast cancer," Applied Surface Science, vol. 332, pp. 308-317, 2015.

[110] Q. Yin, J. Shen, Z. Zhang, H. Yu, and Y. Li, "Reversal of multidrug resistance by stimuli-responsive drug delivery systems for therapy of tumor," Advanced Drug Delivery Reviews, vol. 65, no. 13-14, pp. 1699-1715, 2013.

[111] Q. He, Y. Gao, L. Zhang et al., "A pH-responsive mesoporous silica nanoparticles-based multi-drug delivery system for overcoming multi-drug resistance," Biomaterials, vol. 32, no. 30, pp. 7711-7720, 2011.

[112] C.-H. Lee, S. Cheng, I. Huang et al., "Intracellular pHresponsive mesoporous silica nanoparticles for the controlled release of anticancer chemotherapeutics," Angewandte Chemie-International Edition, vol. 49, no. 44, pp. 8214-8219, 2010.

[113] X. Zhang, F. Li, S. Guo et al., "Biofunctionalized polymerlipid supported mesoporous silica nanoparticles for release of chemotherapeutics in multidrug resistant cancer cells," Biomaterials, vol. 35, no. 11, pp. 3650-3665, 2014.

[114] L. Xiang, P. Su, S. Xia et al., "ABCG2 is associated with HER-2 expression, lymph node metastasis and clinical stage in breast invasive ductal carcinoma," Diagnostic Pathology, vol. 6, article 90, 2011.

[115] D. Damiani, M. Tiribelli, A. Michelutti et al., "Fludarabinebased induction therapy does not overcome the negative effect of ABCG2 (BCRP) over-expression in adult acute myeloid leukemia patients," Leukemia Research, vol. 34, no. 7, pp. 942945, 2010.

[116] S. H. Lee, H. Kim, J.-H. Hwang et al., "Breast cancer resistance protein expression is associated with early recurrence and decreased survival in resectable pancreatic cancer patients," Pathology International, vol. 62, no. 3, pp. 167-175, 2012.

[117] C. H. C. Sukowati, N. Rosso, D. Pascut et al., "Gene and functional up-regulation of the BCRP/ABCG2 transporter in hepatocellular carcinoma," BMC Gastroenterology, vol. 12, article 160, 2012.

[118] Y. Zhao, D. Y. Alakhova, J. O. Kim, T. K. Bronich, and A. V. Kabanov, "A simple way to enhance Doxil ${ }^{\circledR}$ therapy: drug release from liposomes at the tumor site by amphiphilic block copolymer," Journal of Controlled Release, vol. 168, no. 1, pp. 6169, 2013.
[119] E. V. Batrakova, S. Li, A. M. Brynskikh et al., "Effects of pluronic and doxorubicin on drug uptake, cellular metabolism, apoptosis and tumor inhibition in animal models of MDR cancers," Journal of Controlled Release, vol. 143, no. 3, pp. 290-301, 2010.

[120] T. Yamagata, M. Morishita, H. Kusuhara, K. Takayama, H. Benameur, and Y. Sugiyama, "Characterization of the inhibition of breast cancer resistance protein-mediated efflux of mitoxantrone by pharmaceutical excipients," International Journal of Pharmaceutics, vol. 370, no. 1-2, pp. 216-219, 2009.

[121] L. Pan, J. Liu, Q. He, L. Wang, and J. Shi, “Overcoming multidrug resistance of cancer cells by direct intranuclear drug delivery using TAT-conjugated mesoporous silica nanoparticles," Biomaterials, vol. 34, no. 11, pp. 2719-2730, 2013.

[122] L. Jia, Z. Li, J. Shen et al., "Multifunctional mesoporous silica nanoparticles mediated co-delivery of paclitaxel and tetrandrine for overcoming multidrug resistance," International Journal of Pharmaceutics, vol. 489, no. 1-2, pp. 318-330, 2015.

[123] N. Han, Q. Zhao, L. Wan et al., "Hybrid lipid-capped mesoporous silica for stimuli-responsive drug release and overcoming multidrug resistance," ACS Applied Materials and Interfaces, vol. 7, no. 5, pp. 3342-3351, 2015.

[124] D. Spano, C. Heck, P. de Antonellis, G. Christofori, and M. Zollo, "Molecular networks that regulate cancer metastasis," Seminars in Cancer Biology, vol. 22, no. 3, pp. 234-249, 2012.

[125] Q. He, S. Guo, Z. Qian, and X. Chen, "Development of individualized anti-metastasis strategies by engineering nanomedicines," Chemical Society Reviews, vol. 44, no. 17, pp. 6258-6286, 2015.

[126] Y. Chen, P. Xu, H. Chen et al., "Colloidal HPMO nanoparticles: silica-etching chemistry tailoring, topological transformation, and nano-biomedical applications," Advanced Materials, vol. 25, no. 22, pp. 3100-3105, 2013.

[127] Y. Chen, H. Gu, D. S.-Z. Zhang, F. Li, T. Liu, and W. Xia, "Highly effective inhibition of lung cancer growth and metastasis by systemic delivery of siRNA via multimodal mesoporous silicabased nanocarrier," Biomaterials, vol. 35, no. 38, pp. 1005810069, 2014.

[128] M. Egeblad and Z. Werb, "New functions for the matrix metalloproteinases in cancer progression," Nature Reviews Cancer, vol. 2, no. 3, pp. 161-174, 2002.

[129] S. H. van Rijt, D. A. Bölükbas, C. Argyo et al., "Protease-mediated release of chemotherapeutics from mesoporous silica nanoparticles to ex vivo human and mouse lung tumors," ACS Nano, vol. 9, no. 3, pp. 2377-2389, 2015.

[130] R. Roy, J. Yang, and M. A. Moses, "Matrix metalloproteinases as novel biomarkers and potential therapeutic targets in human cancer," Journal of Clinical Oncology, vol. 27, no. 31, pp. 52875297, 2009.

[131] D. Castanotto and J. J. Rossi, "The promises and pitfalls of RNAinterference-based therapeutics," Nature, vol. 457, no. 7228, pp. 426-433, 2009.

[132] Y. Chen, X. Zhu, X. Zhang, B. Liu, and L. Huang, "Nanoparticles modified with tumor-targeting scFv deliver siRNA and miRNA for cancer therapy," Molecular Therapy, vol. 18, no. 9, pp. 1650$1656,2010$.

[133] M. Potente, H. Gerhardt, and P. Carmeliet, "Basic and therapeutic aspects of angiogenesis," Cell, vol. 146, no. 6, pp. 873-887, 2011.

[134] Y. Ohta, Y. Endo, M. Tanaka et al., "Significance of vascular endothelial growth factor messenger RNA expression in primary lung cancer," Clinical Cancer Research, vol. 2, no. 8, pp. 1411-1416, 1996. 
[135] D. Bumcrot, M. Manoharan, V. Koteliansky, and D. W. Y. Sah, "RNAi therapeutics: a potential new class of pharmaceutical drugs," Nature Chemical Biology, vol. 2, no. 12, pp. 711-719, 2006.

[136] X. Liu, A. Situ, Y. Kang et al., "Irinotecan delivery by lipid-coated mesoporous silica nanoparticles shows improved efficacy and safety over liposomes for pancreatic cancer," ACS Nano, vol. 10, no. 2, pp. 2702-2715, 2016. 

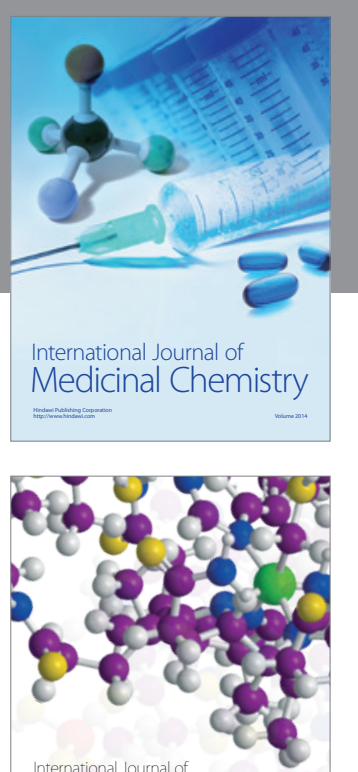

Carbohydrate Chemistry

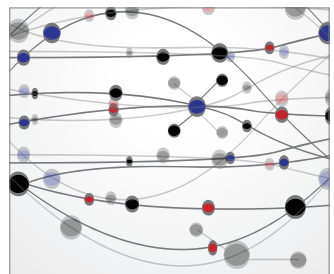

The Scientific World Journal
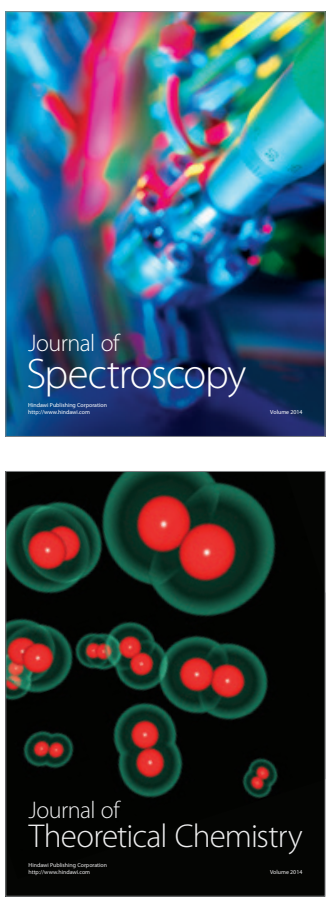
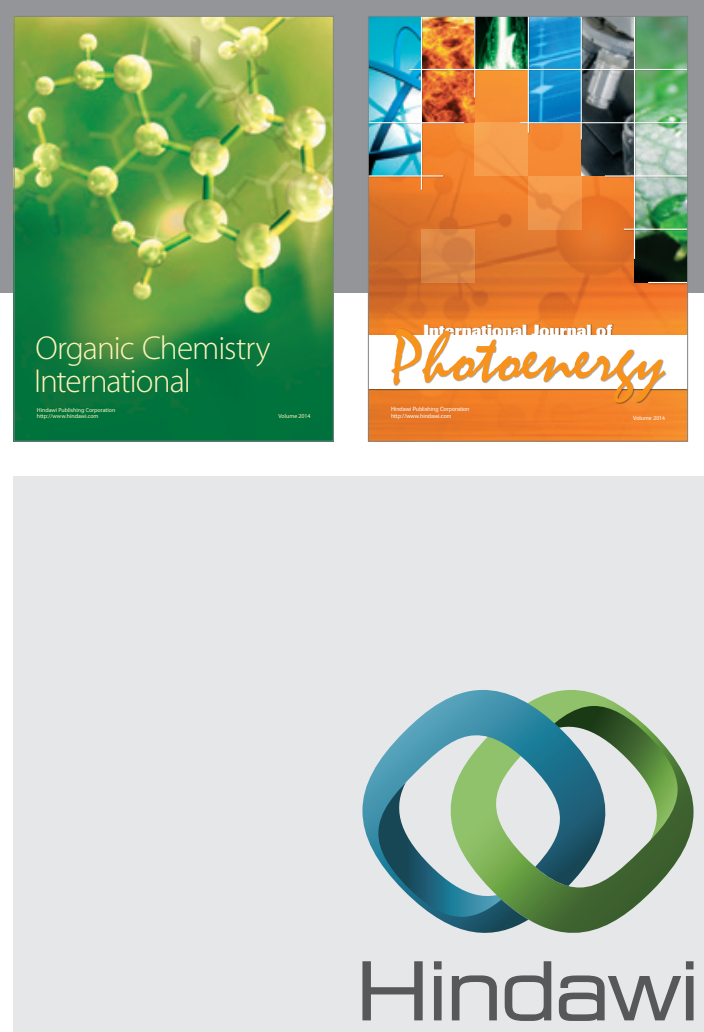

Submit your manuscripts at

http://www.hindawi.com

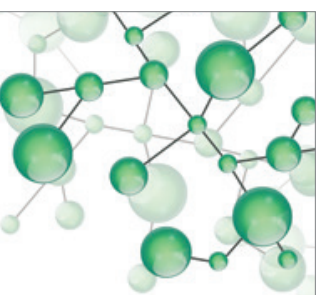

International Journal of

Inorganic Chemistry

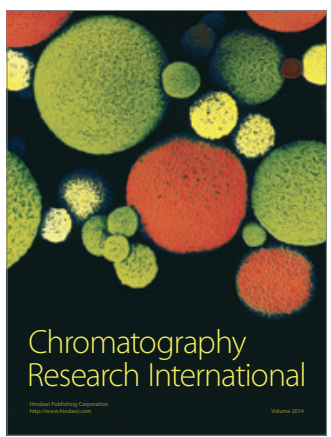

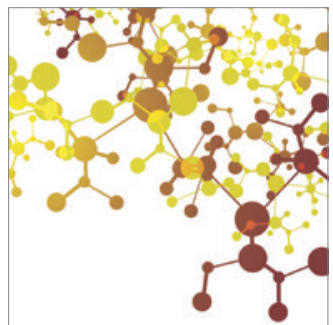

Applied Chemistry
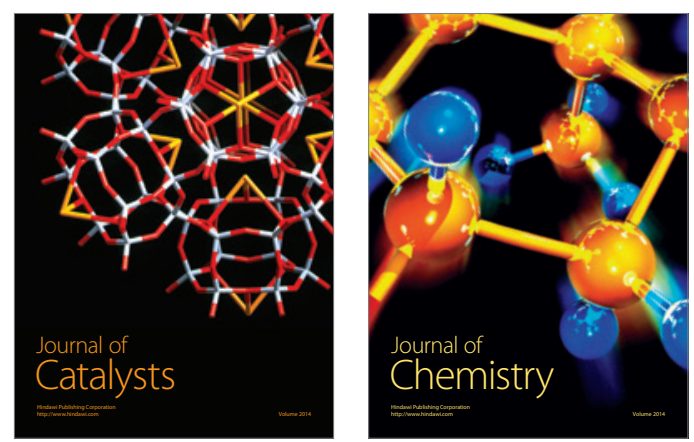
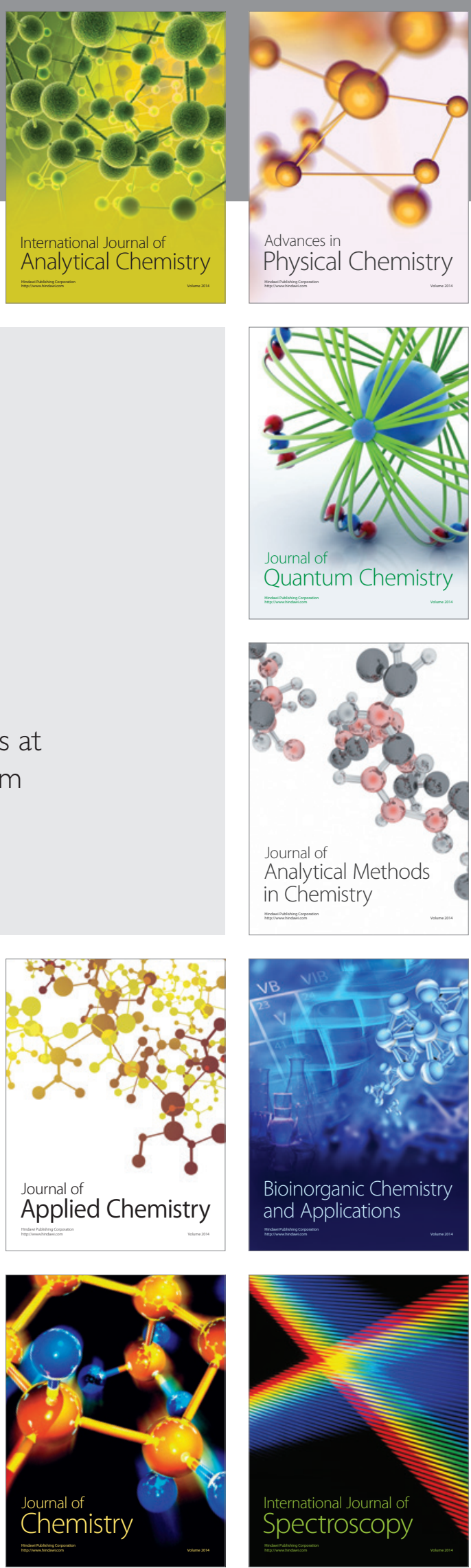